\title{
Optical Testing and Verification Methods for the James Webb Space Telescope Integrated Science Instrument Module Element
}

\author{
Scott R. Antonille*a, Cherie L. Miskey ${ }^{\mathrm{b}, \mathrm{a}}$, Raymond G. Ohl ${ }^{\mathrm{a}}$, Scott O. Rohrbach ${ }^{\mathrm{a}}$, David L. \\ Aronstein $^{\mathrm{a}}$, Andrew E. Bartoszyk ${ }^{\mathrm{a}}$, Charles W. Bowers ${ }^{\mathrm{a}}$, Emmanuel Cofie ${ }^{\mathrm{b}, \mathrm{a}}$, Nicholas R. Collins ${ }^{\mathrm{c}, \mathrm{a}}$, \\ Brian J. Comber $^{\mathrm{d}, \mathrm{a}}$, William L. Eichhorn ${ }^{\mathrm{e}, \mathrm{a}}$, Alistair C. Glasse ${ }^{\mathrm{f}}$, Renee Gracey ${ }^{\mathrm{g}}$, George F. Hartig ${ }^{\mathrm{h}}$, \\ Joseph M. Howard ${ }^{\mathrm{a}}$, Douglas M. Kelly, Randy A. Kimble ${ }^{\mathrm{a}}$, Jeffery R. Kirk, ${ }^{\mathrm{e}}$, David A. Kubalak ${ }^{\mathrm{a}}$, \\ Wayne B. Landsman ${ }^{\mathrm{j}, \mathrm{a}}$, Don J. Lindler ${ }^{\mathrm{k}, \mathrm{a}}$, Eliot M. Malumuth ${ }^{\mathrm{c}, \mathrm{a}}$, Michael Maszkiewicz, Marcia J. \\ Rieke $^{\mathrm{i}}$, Neil Rowlands ${ }^{\mathrm{m}}$, Derek S. Sabatke ${ }^{\mathrm{g}}$, Corbett T. Smith ${ }^{\mathrm{b}}$, J. Scott Smith ${ }^{\mathrm{a}}$, Joseph F. Sullivan ${ }^{\mathrm{g}}$, \\ Randal C. Telfer ${ }^{\mathrm{h}}$, Maurice te Plate ${ }^{\mathrm{n}}$, M. Begoña Vilaa,a, Gerry D. Warner ${ }^{\mathrm{m}}$, David Wright ${ }^{\mathrm{b}, \mathrm{a}}$, \\ Raymond H. Wright ${ }^{0, a}$, Julia Zhou ${ }^{\mathrm{m}}$, Thomas P. Zielinski ${ }^{\mathrm{a}}$ \\ aNASA’s Goddard Space Flight Center, 8800 Greenbelt Rd., Greenbelt, Maryland \\ bStinger Ghaffarian Technologies, 7701 Greenbelt Rd \#400, Greenbelt, Maryland \\ 'KBRwyle, 1290 Hercules Avenue, Houston, Texas \\ ${ }^{\mathrm{d} C}$ Comber Thermal Solutions, 8367 Silver Trumpet Dr, Columbia, Maryland \\ ${ }^{\mathrm{e}}$ Genesis Engineering Solutions, Inc., 4501 Boston Way, Lanham, Maryland \\ fUK Astronomy Technology Centre, Edinburgh, United Kingdom \\ 'Ball Aerospace \& Technologies Corporation, 1600 Commerce St., Boulder, Colorado \\ ${ }^{\text {h}}$ Space Telescope Science Institute, 3700 San Martin Dr., Baltimore, Maryland \\ iSteward Observatory, University of Arizona, Tucson, Arizona \\ jADNET Systems, Inc., 6720B Rockledge Dr., Suite 504, Bethesda, Maryland \\ ${ }^{k}$ Sigma Space Corporation, 4600 Forbes Blvd., Lanham, MD 20706 \\ lCanadian Space Agency, 6767 route de L’Aéroport, Saint-Hubert, Canada \\ moneywell Aerospace, 303 Terry Fox Dr., Suite 100, Ottawa, Canada \\ ${ }^{n}$ European Space Agency - STScI, 3700 San Martin Dr., Baltimore, Maryland \\ ${ }^{\circ}$ AK Aerospace Technology Corporation, 12970 Brighton Dam Road, Clarksville, Maryland
}

\begin{abstract}
NASA's James Webb Space Telescope (JWST) is a 6.6m diameter, segmented, deployable telescope for cryogenic IR space astronomy ( 40K). The JWST Observatory includes the Optical Telescope Element (OTE) and the Integrated Science Instrument Module (ISIM) that contains four science instruments (SI) and the fine guider. The SIs are mounted to a composite metering structure. The SI and guider units were integrated to the ISIM structure and optically tested at the NASA Goddard Space Flight Center as a suite using the Optical Telescope Element SIMulator (OSIM). OSIM is a full field, cryogenic JWST telescope simulator. SI performance, including alignment and wavefront error, were evaluated using OSIM.

We describe test and analysis methods for optical performance verification of the ISIM Element, with an emphasis on the processes used to plan and execute the test. The complexity of ISIM and OSIM drove us to develop a software tool for test planning that allows for configuration control of observations, associated scripts, and management of hardware and software limits and constraints, as well as tools for rapid data evaluation, and flexible re-planning in response to the unexpected. As examples of our test and analysis approach, we discuss how factors such as the ground test thermal environment are compensated in alignment.

We describe how these innovative methods for test planning and execution and post-test analysis were instrumental in the verification program for the ISIM element, with enough information to allow the reader to consider these innovations and lessons learned in this successful effort in their future testing for other programs.
\end{abstract}

Keywords: JWST, ISIM, testing, verification, requirements, optical testing 


\section{INTRODUCTION}

NASA's James Webb Space Telescope (JWST) is a 6.6m diameter, segmented, deployable telescope for cryogenic IR space astronomy, with hardware operating near $\sim 40 \mathrm{~K}$. The JWST Observatory architecture includes the Optical Telescope Element (OTE) and the Integrated Science Instrument Module (ISIM) element. The Integrated Science Instrument Module (ISIM) is comprised of four Science Instruments (SI's), the Fine Guidance Sensor (FGS), and the ISIM structure, to which each of the instruments is mounted. ISIM was tested at NASA's Goddard Space Flight Center in three separate, optical, cryogenic campaigns with the aid of a highly configurable OTE SIMulator (OSIM). In this paper, we summarize the success of the innovations associated with the planning, architecture, and working components of the ISIM optical verification campaign that evolved from the effort of many workers over nearly a decade. Additional information on the SIs ${ }^{1,2,3,4}$, an overview of ISIM $^{5}$, the overall test campaign ${ }^{6}$, and a description of OSIM $^{7}$ are found in the literature.

\subsection{Role of ISIM Cryogenic-Vacuum Testing for JWST}

The ISIM cryogenic-vacuum (CV) test campaign is an essential component of ensuring the success of the JWST observatory. Although the JWST OTIS (i.e., OTE \& ISIM) assembly will undergo an end-to-end, cryogenic optical test NASA's Johnson Space Flight Center (JSC), severe ground-test effects and the infeasibility of constructing a full-aperture, cryogenic, optical test stimulus constrained the design and capability for testing at that level of assembly $y^{8,9}$.

Thus, the ISIM CV tests were more than a straightforward check on alignment and performance: ISIM-to-nominal-OTEinterface alignment, SI co-alignment, SI co-alignment stability, SI wavefront error verification and calibration, and other SI performance verifications and characterizations derived from this ISIM-Element cryo-vac test campaign play a direct role in the final, pre-launch Observatory verification and input to commissioning during flight. Aspects of the observatory commissioning program are reliant on the alignment and wavefront error calibrations from ISIM CV testing. Moreover, where needed, the ISIM CV tests assisted with SI- or sub-system-level optical verifications, science data-related characterizations, or checks that required an accurate, high-fidelity, simulated point source or high-quality flat field. Early in the planning process, it became clear that the ISIM optical performance verification campaign would be both critical and complex.

\subsection{Evolution of ISIM Optical Performance Verification and Cryogenic-Vacuum Testing}

If evolution can be considered the accumulation of successful adaptations to external pressures, then the innovations in the planning, architecture, and toolset development for the ISIM optical verification campaign is evolution driven by the development of the JWST Science Instruments, ISIM, and the JWST program as a whole. We hope that our development work and lessons learned from this evolutionary process find application on future projects elsewhere in the community.

We realized from the start that ISIM optical performance verification and the associated testing would be complex, given the number and diversity of requirements, the number and different types of optical channels, etc. However, as the project progressed and testing neared, cleanly-laid plans were replaced with the increased complexity of reality. It was clear our plans and procedures would have to evolve. As the observatory became better defined and analyzed, new characterizations and verifications in the ISIM CV test became necessary. Additionally, our optical verification planning necessarily occurred in parallel with the build and component testing of the SIs, OSIM, and other major ISIM sub-systems critical to the test and verification effort. As we made initial plans, instruments were redesigned, OSIM capabilities evolved, and command and data transfer interfaces were in flux. New questions arose, and new tests were required to answer them. It was from this environment that the fundamental methodology, test architecture, and software toolsets described here were conceived and defined. Once the basic components were conceived, there were plenty of twists and turns, lessons learned, and adaptions made; however, it was the evolution of the methodology, architecture, and software in an uncertain and complex environment that helped pre-adapt our approach to further change.

\subsection{ISIM Optical Test and Verification: Methodology, Software, Architecture, and Process}

This paper will not endeavor to narrate the twists and turns of each development and adaptation. What is presented from this point forward is the final product: the methodologies that allowed us to break down the performance verification and characterization requirements into required data and an analysis flow, the tools that enabled us to turn those required observations into executable tests, the formal test procedure structure that enabled us to take full advantage of the processes we used to maintain tight quality assurance (QA) and configuration management (CM) during optical testing despite unexpected results, the quick turnaround analysis that allowed testing to proceed efficiently, and, finally, the organized handoff of data products to analysts that completed the analysis flow to provide the verification and characterizations required. 
We describe methods for optical performance verification of the ISIM Element, with an emphasis on the processes used to plan and execute the test, and the optical analysis to verify requirements. Figure 1-1 provides an overview of the workflow

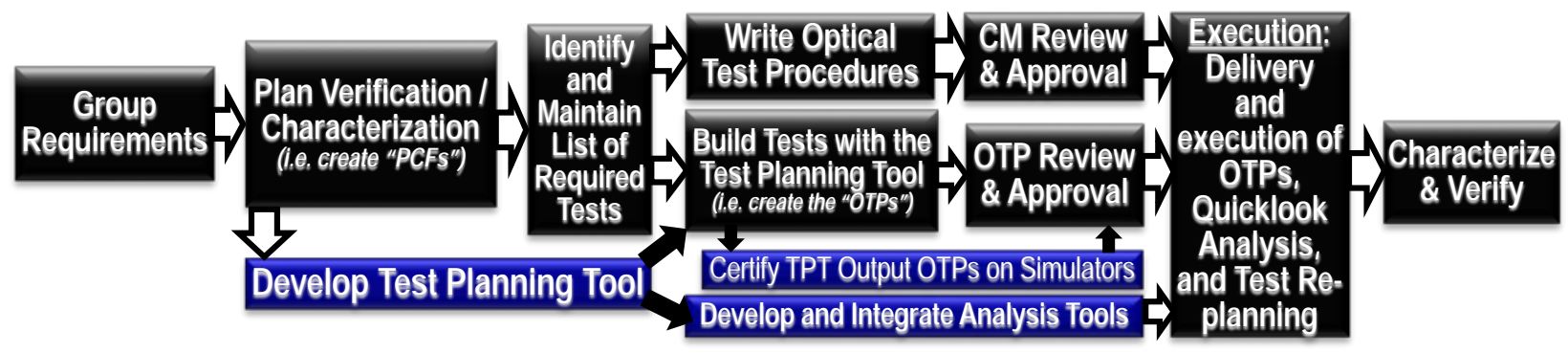

Figure 1-1 Overview of the ISIM Element Optical Performance Verification Workflow

\section{MOVING FROM REQUIREMENTS TO PERFORMANCE VERIFICATION PLANS}

The first challenge in ISIM optical performance verification was developing a methodology for organizing requirements from many documents into groups of optically similar material and then fabricating a manageable set of plans for performance characterization and verification. To capture the logic and processes required to verify optical performance requirements from test data and other sources, we developed Performance Characterization Flows (PCFs). These PCFs ultimately set test requirements, directed the development of the tests, and defined how those tests will be analyzed to provide the characterization or verification required.

The results of this many-year effort are documented in the ISIM Optical Performance Verification Plan ${ }^{10}$. The compilation of this document is a product of years of evolution, the effort of many contributors and reviewers, and the effort of the author to keep the content up to date and manageable for a reader. The content and format of each section of the document were a product of continual adaptation and careful thinking.

The methodology, products, and lessons learned in the many year effort to transform a uniquely complex set of requirements into a documented characterization and verification plan is presented here.

\subsection{ISIM Requirement Structure and ISIM Optical Requirements}

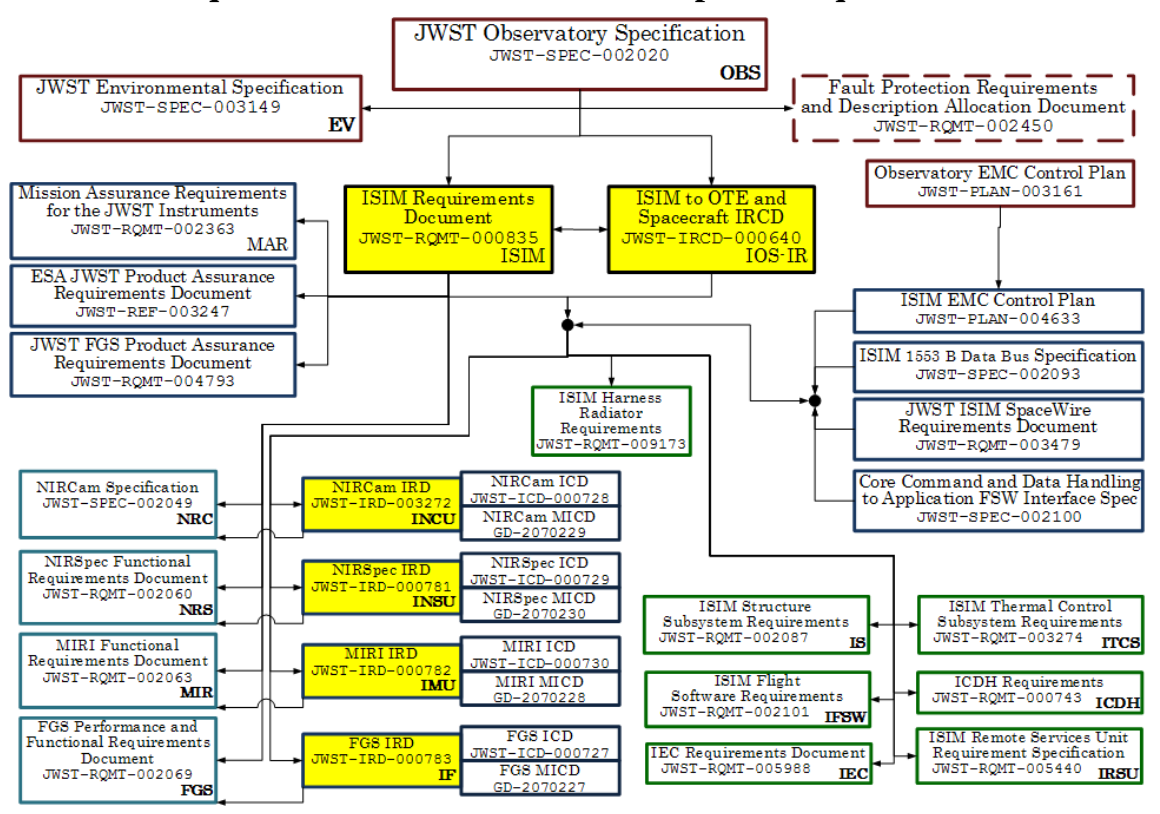

Figure 2-1 The controlling engineering requirements documents for ISIM shown as a flow chart. This diagram shows engineering requirements and does not extend to mission and science requirements documents. the inevitable growth of complexity.
For example, we developed and implemented ISIM CV tests of SI performance trends, sub-system requirements
The controlling requirements documents are shown in Figure 2-1. Systems engineers grouped these requirements by discipline. The $>149$ ISIM requirements were assigned to optical engineering and are derived from the six documents highlighted in yellow in Figure 2-1.

This diagram provides insight into the technical and disciplinary interdependency of delivering ISIM and the myriad teams whose efforts needed to be communicated and coordinated to be successful. One essential example of coordination in the optics discipline was between the optics team and SI teams as we incorporated their up-to-date feedback directly into ISIM-level CV test plans.

An experienced eye may also view this requirement linkage and identify the inevitable growth of complexity. 
requiring final verification, on-going performance investigations from sub-system-level testing, and, in some cases, characterizations of SI hardware swap-outs between tests.

In the following section (Section 3) the impact of the evolving complexity and coordination required will have relevance to our test development and implementation approach. However, for the remainder of this section, the focus is on the ISIM optical requirements and our development of the Optical Performance Verification Plan.

\subsection{Identification and Grouping of ISIM Optical Requirements}

The 149 optical requirements were organized into six Optical Performance Requirement Groups (OPRG):

- OPRG 1 - ISIM Capabilities and Function

$\hookrightarrow$ This group covers basic ISIM optical capabilities and optical function. The requirements assigned to this group are those that require basic functionality from the ISIM (sometimes referred to as "motherhood" requirements) or require the input of many disparate pieces of performance information for verification.

- OPRG 2 - Wavefront Error and Focus

$\hookrightarrow$ This group holds the requirements that specify the amount of wavefront error and focus error that the ISIM element is allowed. This is also the group that contains the calibration requirements for the multi-instrument, multi-field (MIMF) wavefront sensing algorithm.

- OPRG 3 - Pupil Alignment

$\hookrightarrow$ This group contains the requirements that specify how much pupil alignment error the ISIM element is allowed with respect to the nominal OTE exit pupil. The pupil errors of interest are lateral shear and pupil rotation (also sometimes referred to as "clocking").

- OPRG 4 - Field of View, Vignetting, and Stray Light

$\hookrightarrow$ Requirements relating to the SI fields of view, vignetting, and stray light, and also additional requirements that specify the absolute pointing of ISIM.

- OPRG 5 - Co-Boresight Stability

$\hookrightarrow$ These requirements specify how much the SI pointing is allowed to change over different periods of time on-orbit, which we relate to the changing thermal conditions of the ISIM.

- OPRG 6-WFS\&C Component Verification

$\hookrightarrow$ The requirements assigned to this group are those related to the performance of wavefront sensing and control (WFSC) components in the NIRCam instrument. They include requirements that were verified at the NIRCam-level, but require additional test data at the ISIM-level.

In Figure 2-2, we show a sample of how the optical requirements levied on ISIM were organized into requirement groups in the Optical Performance Verification Plan, where each requirement is identified with its unique identifier (ID) number.

OPRG Requirements

\begin{tabular}{l|l|}
$\begin{array}{l}\text { 1: ISIM Capabilities } \\
\text { and Function }\end{array}$ & $\begin{array}{l}\text { ISIM-152, ISIM-852, ISIM-252, ISIM-253, ISIM-1290, ISIM-1363, ISIM-1364, ISIM-1365, ISIM-153’, ISIM-264, ISIM-320, IOS-IR- 725’, IOS- } \\
\text { 2: WFE and Focus }\end{array}$ \\
& ISIM-668, ISIM-1259, ISIM-213, ISIM-223, ISIM-851, ISIM-1034, ISIM-688, ISIM-1035, ISIM-319, IOS-IR-387, IOS-IR-5778, IOS-IR-5960, \\
& IOS-IR-5962, IOS-IR-5964, IOS-IR-5772, IOS-IR-5777, IOS- IR-5817, IOS-IR-5821, IOS-IR-5822, IOS-IR-5824, IOS-IR-5825, \\
& IOS-IR-5826, IOS-IR-5829, IOS-IR-5831, IOS-IR-5833, IOS-IR-5835, IOS-IR-5837, IOS-IR-5839, IOS-IR-5841, IOS-IR-5843, IOS-IR-5845, \\
\hline 3: Pupil Alignment & $\begin{array}{l}\text { ISIM-1036, IOS-IR-5848, IOS-IR-5849, IOS-IR-5851, IOS-IR-725”, } \\
\text { 2156, IMU-2300, INSU-1819-IR-5801, IOS-IR-5910, IOS-IR-5798, IOS-IR-5799, IOS-IR-5790, IOS-IR-5803, IOS-IR-5804, IF-114, IMU- }\end{array}$ \\
\hline
\end{tabular}

Figure 2-2 Excerpt from a table showing requirement IDs grouped under each OPRG.

\subsection{Performance Characterization Flows}

For each requirement group (i.e., OPRG), multiple Performance Characterization Flows (PCFs) are developed to address all the requirements associated with that group. To make that association clear, a hierarchical structure was constructed to maintain associations between requirement groups and PCFs that covered subsets of requirements in that group. For example, OPRG 3, requirements relating pupil alignment, contains PCFs 3.1 - 3.3, and the sum of all PCFs associated with one optical performance requirement group (PCFs 3.1, 3.2, and 3.3) addresses all the requirements in OPRG 3.

The PCF organizational approach had many advantages. The first benefit was that, given a PCF ID, one could instantly understand the requirement type addressed and, therefore, the nature of the associated tests, data, and analysis. That is, the PCF ID nomenclature provided a meaningful shorthand aid that helped us discuss and organize the test. This hierarchical organizational scheme was further extended to test development and execution and the benefits of that are discussed later in this paper.

More importantly, the PCF organization structure eliminated the necessity of one-to-one linkages between requirements and verification/test plans. That allowed for two important efficiencies.

First, if a large group of requirements could be verified from a common dataset, these could be all grouped into one PCF. Not only does this simplify documentation, it immediately identifies where efficiency can be gained in test plans. 
Additionally, where characterization or verification share model data, test data, data reduction and analyses, the organization improves coordination between analysts and improves the accuracy and consistency of a-priori information and data used in the characterization/verification process.

The PCF's are documented in the Optical Performance Verification Plan and each contains:

1. A brief summary of what the PCF addresses

2. A summary of the requirements addressed ${ }^{1}$

3. An overview table of the SI exposure configurations required to verify the requirements and an associated rationale, description, or narrative explaining those exposure configurations (Figure 2-3).

\begin{tabular}{|c|c|c|c|c|}
\hline \multicolumn{5}{|c|}{ Imaging Configuration Overview (sample) } \\
\hline Instrument & $\begin{array}{c}\text { Pupil(P)/ } \\
\text { Filter (F)/ } \\
\text { Grating (G) }\end{array}$ & $\begin{array}{c}\text { OSIM } \\
\text { Source }\end{array}$ & $\begin{array}{c}\text { OSIM } \\
\text { Pupil }\end{array}$ & Field Point \\
\hline $\begin{array}{c}\text { NIRCam A/B } \\
\text { (PIL) }\end{array}$ & $\begin{array}{c}\text { P: Imaging } \\
\text { F: F187N }\end{array}$ & $\begin{array}{c}\text { Super- } \\
\text { Continuum }\end{array}$ & PAR \#1 & PIL Center \\
\hline $\begin{array}{c}\text { NIRCam A/B } \\
\text { (PIL) }\end{array}$ & $\begin{array}{c}\text { P: Imaging } \\
\text { F: F187N }\end{array}$ & $\begin{array}{c}\text { Super- } \\
\text { Continuum }\end{array}$ & $\begin{array}{c}\text { Hartmann- } \\
\text { Distortion }\end{array}$ & PIL Center \\
\hline MIRI Imaging & F: FLENS & LD155 & PAR \#2 & MIRIM PAL \\
\hline $\begin{array}{c}\text { NIRSpec + } \\
\text { OSIM PIM }\end{array}$ & PAR & LD064 & PAR \#1 & Center \\
\hline $\begin{array}{c}\text { FGS-Guider + } \\
\text { OSIM PIM }\end{array}$ & PAR & $\begin{array}{c}\text { FGSA } \\
\text { LD064 }\end{array}$ & Open & TMA Center \\
\hline $\begin{array}{c}\text { NIRISS + } \\
\text { OSIM PIM }\end{array}$ & $\begin{array}{c}\text { P: CLEARP } \\
\text { F: CLEAR }\end{array}$ & LD064 & PAR \#1 & ISIM 14
\end{tabular}

\section{Test Imaging Rationale}

The fundamental images of this test are those with the OSIM PIM using the OSIM PAR \#1, such that both the SI PAR pattern and the OSIM PAR pattern are present in the image, permitting a measurement of the center, rotation, and scaling of each PAR. To get the cleanest possible look at the SI PARs, PIM images are also acquired with the OSIM Open pupil. This will allow a clearer look at not only the crosshairs but also the SI PAR fiducials, which could be hidden by the OSIM par. Also, if there are any irregularities in the PAR image due to surface quality, having such images will give us the best opportunity to account for this, for example by dividing the OSIM PAR\#1 image by the Open image. Images of the point source on the SIs to establish field are not included, as the OSIM pointing errors are considered to be insignificant for this test but the uncertainty in pupil wander should be

Figure 2-3 An excerpt showing exposure configuration and associated rationale text.

This section was critical in producing and early, optic-centric overview of what optical testing was necessary including what data collection or quality aspects were critical to the verification goals.

4. The verification description which consists of:

a. The Data Required: A comprehensive table of all additional information required to perform the verification(s) such as models, sub-system test results, and ISIM CV test derived products (Figure 2-4).

\begin{tabular}{|c|c|c|c|}
\hline \multicolumn{4}{|c|}{ PRIMARY INPUTS TO VERIFICATION } \\
\hline \multicolumn{4}{|c|}{ Required “A Priori” Input for Main Analysis } \\
\hline Input & Data & Source & Notes \\
\hline 1 & OSIM Pupil Motion Calibration & OSIM calibration & \\
\hline 2 & OSIM PAR 1 and 2 V-Coord Calibration & OSIM calibration & \\
\hline 3 & OSIM PAR 1 and 2 Fiducial Calibration & OSIM calibration & \\
\hline 4 & SI Pupil Reference Features Characterization & SI EIDP & PAR feature metrology \\
\hline 5 & SI Pupil References Placement Knowledge & SI EIDP & offsets wrt SI pupil \\
\hline 6 & Ground-to-Orbit Pupil Change & ISIM STOP Modeling & \\
\hline \multicolumn{4}{|c|}{ Test Data and Telemetry } \\
\hline 8 & NIRCam A and B PIL Detector Data & NIRCam Perf. Char. & \\
\hline 9 & MIRI Alignment Lens Detector Data & MIRI Perf. Char. & \\
\hline 10 & SI Images of OSIM PAR source pointing & SI Perf. Char. & PAR field point used \\
\hline 11 & SI PAR + OSIM PAR Measurements w/ PIM & SI Perf. Char. & \\
\hline \multicolumn{4}{|c|}{ ISIM Metrology } \\
\hline 13 & SI Bench alignment in 1g to V-coords & ISIM Metrology & \\
\hline \multicolumn{4}{|c|}{ Other Critical Inputs for Analysis } \\
\hline 14 & OTE Optical Model & IOS OTE model & \\
\hline 15 & SI Optical Model & SI EIDP & \\
\hline
\end{tabular}

Figure 2-4 An excerpt showing a list of required data.

- This allowed test precedents test dependencies to be mapped out early in the test development phase.

- When verification relied upon modeling (such as STOP results) or sub-system End Item Data Package (EIDP) deliverables, this list was a catalyst to ensure that the precise model or dataset necessary in the verification process was known, acquired when it became available, and became the uniform input across any derivative analyses.

b. An Outline of the Verification Process: A simplified process outline primarily showing how the previously listed data/information would be utilized to create verification products (Figure 2-5).

\begin{tabular}{|c|c|c|c|c|c|c|}
\hline \multicolumn{7}{|c|}{ VERIFICATION PROCESS OUTLINE } \\
\hline Step & Input & Quick Description & Process or Technique & Description & Output & Uncertainty \\
\hline B & $\begin{array}{r}8,9 \\
10,11 \\
\end{array}$ & Locate PAR & $\begin{array}{l}\text { Image registration, } \\
\text { interactive fitting }\end{array}$ & $\begin{array}{l}\text { Image analysis to calculate PAR and pupil } \\
\text { reference positions in local coordinates }\end{array}$ & $\begin{array}{l}\text { PAR and Pupil Reference } \\
\text { Locations in Detector Coordinates }\end{array}$ & Pupil fiducial location error \\
\hline C & $\begin{array}{l}3,4 \\
10,11\end{array}$ & Scale PAR's & $\begin{array}{l}\text { Ratio imaged fiducial } \\
\text { separation vs known }\end{array}$ & $\begin{array}{l}\text { Calculate pupil mag from fiducial } \\
\text { calibration }\end{array}$ & $\begin{array}{l}\text { Image scale for PAR features in } \\
\text { OTE exit pupil space }\end{array}$ & $\begin{array}{l}\text { Repeatability error estimates come from re- } \\
\text { insertion loops for the OSIM PAR and the } \\
\text { SI pupil references }\end{array}$ \\
\hline D & & $\begin{array}{c}\text { Compare PAR } \\
\text { Locations }\end{array}$ & $\begin{array}{l}\text { Solve for PAR coordinate } \\
\text { transform }\end{array}$ & $\begin{array}{l}\text { Calculate relative error in } \mathrm{mm} \text { and radians } \\
\text { between SI pupil references and OSIM } \\
\text { pupil references }\end{array}$ & $\begin{array}{l}\text { Pupil shear and roll error between } \\
\text { SI pupil references and OSIM } \\
\text { pupil references }\end{array}$ & $\begin{array}{l}\text { Repeatability error estimates come from re- } \\
\text { insertion loops for the OSIM PAR and the } \\
\text { SI pupil references }\end{array}$ \\
\hline
\end{tabular}

${ }^{1}$ Initially, we had included a rationale, discussion, and interpretation of each requirement in a PCF at the start of the PCF. Some requirements necessitated a lot of discussion about the initial rationale in order to formulate an interpretation of that requirement that was relevant to what could be measured or analyzed. These rationales and interpretations became cumbersome to carry in each PCF, and they were moved into an appendix of the ISIM Optical Performance Verification Plan. This was an important adjustment to keep the PCF focused on the verification process itself. 


\begin{tabular}{|c|c|c|c|c|c|c|}
\hline \multirow{2}{*}{\multicolumn{7}{|c|}{$\begin{array}{c}\text { VERIFICATION PROCESS OUTLINE } \\
\text { Description }\end{array}$}} \\
\hline & & & & & Output & Uncertainty \\
\hline $\mathrm{E}$ & $\begin{array}{l}2, \mathrm{~b}, \\
12,14\end{array}$ & Calculate Pupil Error & $\begin{array}{l}\text { Apply } \Delta \text { (YAK-pupil) } \\
\text { knowledge }\end{array}$ & $\begin{array}{l}\text { SI pupils and the nominal OTE exit pupil } \\
\text { in V-coords }\end{array}$ & $\begin{array}{l}\text { so Pupul trror in Ground } \\
\text { Environment at Tested Field Points }\end{array}$ & $\begin{array}{l}\text { USIM PAK Calibration, YAK/YYupII } \\
\text { Reference Calibration }\end{array}$ \\
\hline G & 6 & $\begin{array}{c}\text { Remove Ground } \\
\text { Effects }\end{array}$ & STOP Modeling & $\begin{array}{l}\text { Remove pupil shear and roll errors due to } \\
\text { Test Environmental Effects }\end{array}$ & $\begin{array}{c}\text { On-Orbit SI Pupil Errors at Test } \\
\text { Field Point }\end{array}$ & Error propagation through STOP modeling \\
\hline $\mathrm{H}$ & $15, \mathrm{~F}$ & Error at FOV Center & Optical Modeling & $\begin{array}{l}\text { Calculate On-Orbit SI Pupil Errors at SI } \\
\text { Center Field Point }\end{array}$ & $\begin{array}{c}\text { On-Orbit SI Pupil Error at SI } \\
\text { Center Field Point }\end{array}$ & \\
\hline
\end{tabular}

Figure 2-5 Excerpt showing an outline of the verification process for a requirement.

- This provided a map of how the data required was fit together through analysis to perform one or more verifications.

- It also highlighted how component-level uncertainties contributed to uncertainties in the final analysis products. This not only assists in linking these plans to the test error budget, but also sheds light on whether the end product uncertainty is dominated by ISIM testing or other uncertainty factors in the verification flow. The associated test design can then be informed appropriately.

c. Output products: A table that cross-references the verification outputs in the analysis outline with specific requirements or any other use, such as a crosscheck, for the end analysis products (Figure 2-6).

\begin{tabular}{|c|l|c|c|c|}
\hline \multicolumn{5}{|c|}{ VERIFICATION OUTPUTS AND CROSSCHECKS } \\
\hline Product Name & \multicolumn{1}{|c|}{ Description } & Step & Utility & Deliver to: \\
\hline NIRCam Pupil Error & On-Orbit NIRCam pupil shear in all NIRCam Channels & I & IOS-IR-5798 & ISIM Systems \\
\hline Non-NIRCam Pupil Error & On-Orbit non-NIRCam pupil shear in all instrument channels & I & IOS-IR-5799 & ISIM Systems \\
\hline SI Pupil Roll & On-Orbit SI pupil roll/rotation error in all instrument channels & I & IOS-IR-5790 & ISIM Systems \\
\hline $\begin{array}{c}\text { Optical Model Cross- } \\
\text { Check }\end{array}$ & $\begin{array}{l}\text { Comparison of as-built model prescription of pupil wander at } \\
\text { PAR field point versus measured }\end{array}$ & F & Crosscheck & $\begin{array}{c}\text { ISIM Optical } \\
\text { Analysis }\end{array}$
\end{tabular}

Figure 2-6 Excerpt showing a list of output products.

- In cases where analysis products from one PCF were required data for another PCF, it allowed us to map test dependencies.

d. Verification summary: A narrative explanation outlining the data analysis and how it is used to produce pass/fail criteria or performance characterization deliverables (Figure 2-7).

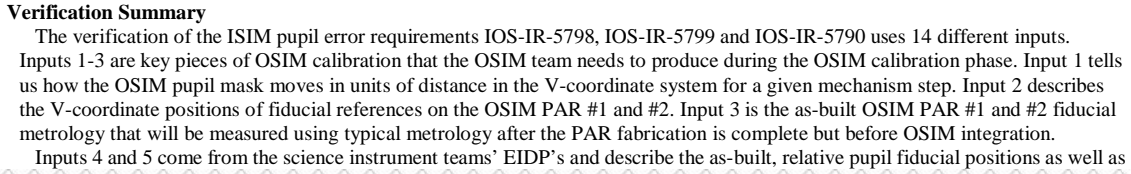

Figure 2-7 Excerpt showing a verification summary.

In a separate section, the Optical Performance Verification Plan addresses the optical requirements with an explanation of how they and their rationale have been translated into metrics that we can characterize and/or verify through test and analysis. The verification outline and summary conclude the PCF by demonstrating how that metric is produced and evaluated through test and analysis.

\subsection{Benefits of the ISIM Performance Verification Plan and PCF Architecture}

In the section above, we emphasized the benefits of the organization, architecture, and content of the PCFs on subsequent test planning efforts. These come into play after the PCFs have reached a fairly mature state. The process of developing the PCFs, however, held just as many benefits.

The critical and difficult first step in generating the Optical Performance Verification Plan was to start from a set of assigned requirements and translate them into measurable metrics. For example, simple sounding requirements might need more concrete physical interpretations to be verified, and once a specific physical property is identified, it can end up being elusive to verify with the available measurement apparatus. This step was initiated by handing off the requirements to the prospective optical system engineers and analysts who would be charged with their verification. As they, in conjunction with key stakeholders in the requirements, translated requirements into metrics accessible through test and/or analysis, the progress on the PCFs could begin effectively.

With that first step complete, the development of the PCFs have an end goal, and the content of the PCFs can be developed and reviewed with that goal in mind. That goal also illuminated additional detail required in the verification analysis. As those details were fleshed out, the extent of the inputs necessary for the verification process become better defined, including, but not limited to, the required test data set. Additionally, where verification analysis relied on results from modeling, the required modeling tasks become more clearly defined.

In reality this process was iterative, non-linear, and always in flux. Yet this development process initiated more in-depth thought about verification that allowed the ISIM optical team to forge ahead without significant reliance on sub-system testing or delivery. In contrast, test development is very difficult without detailed knowledge of mature sub-systems that constitute the whole article under test. 
An additional benefit to the process and format used in the Optical Performance Verification Plan is that it provided a single structure that could be used by many developers, contributors, reviewers, and readers involved. It enabled many individual contributions to be complied into a document with a consistent structure. That structure became familiar to contributors and reviewers alike, allowing the document to be used more effectively.

\section{THE TEST PLANNING TOOL AND QUICKLOOK ANALYSIS SOFTWARE}

\subsection{The Test Planning Tool}

\section{Motivation and Development of the Test Planning Tool Concept}

The Test Planning Tool (TPT) development started in late 2010 to address the challenge of planning many hundreds of optical tests for several cryo-vac campaigns. The standing protocol at the time would be to write one or more scripts for each test, certify the scripts in a hardware simulation lab, and then execute those scripts when called for in the optical test procedures. It quickly became obvious that this would be infeasible for ISIM Optical CV testing.

At the outset of the TPT development effort, our team members looked into any precedents set by past optical test programs (particularly in the aerospace field). We found no precedent for planning or running an optical test campaign that approached the combined complexity of OSIM and ISIM. Nor did we find any optical test program of similar complexity that was subject to the rigor of mission assurance QA and CM requirements that are associated with a JWSTclass mission. The general pattern in the optical test programs we investigated was that if the program was at a point where strict QA and CM requirements were enforced, optical testing tended to be limited and simple. If an optical test campaign was more complicated, then there was considerably less emphasis on QA, CM, safety, and traceability. I believe that this reflects the reality of optical testing: as an optical test becomes more complicated, the number of deviations from and tweaks to the pre-planned tests grow so quickly that strict adherence to QA/CM procedures becomes a serious impediment to testing.

The ISIM CV optical test campaign was certainly going to be complicated, but we also had to comply with the JWST mission assurance QA and CM requirements. The Test Planning Tool had to enable a test planner to make the necessary deviations and tweaks to the optical tests, maintain QA/CM protocols, and do so without introducing additional work for the test planner or causing significant test delays.

Developing specialized scripts for each test was a logistical impossibility and was abandoned early. The optical tests needed to be architected by the optics personnel who were integral in creating the Performance Characterization Flows (PCFs) as discussed in Section 2.3. However, writing the command scripts to perform the test had to be done by the ISIM operations team. This approach may have been feasible if only a handful of scripts had to be written, but given the magnitude of the test campaign, neither the Optics nor Operations teams had the manpower available to coordinate the creation of so many specialized scripts. Furthermore, the development of the tests was an extended effort, and if creating the scripts could not be started until the test design was finalized, then we would simply run out of time. There needed to be a division of labor between the Optics teams constructing the tests and the Operations team that would implement those tests. Furthermore, it was essential that each team could make progress in parallel.

The overwhelming majority of optical tests required by the PCFs in the Performance Verification Plan could be accomplished by repeating a common schema which we refer to as an "activity": configure ISIM and OSIM, trigger the illumination and exposure, and finish by "cleaning up" any configuration or settings that should not persist to the next activity. Although there were a near infinite set of unique and valid configuration and exposure parameters, the structure and execution of an activity could conform to a well-defined flow. The ISIM Optics Test Planning Team and ISIM Operations team realized that this schema could form the basis of a test planning and execution system that could

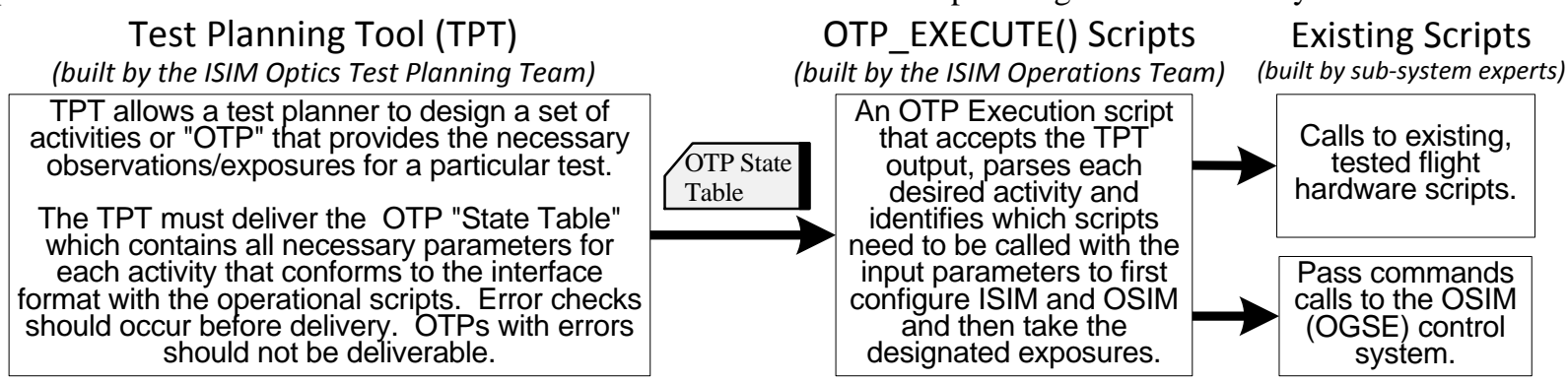

Figure 3-1 Framework for Implementing a Test Planning Tool to Specify Optical Test Operations

accommodate almost any valid optical test. Additionally, this schema suggested a division of labor that would allow for optimal parallel development of the optical tests and the supporting scripts. As depicted in Figure 3-1, the Test Planning 
Team could develop the Test Planning Tool to construct sequences of activities in what became known as an "OTP²." An OTP can exist in various formats, but it always refers to a sequence of test activities. The TPT could then generate an OTP state table that lists all of the necessary parameters in a strictly controlled format. The Operations team could then construct a script capable of parsing that file, and in a well-defined sequence, pass the requested parameters to existing ISIM and OSIM routines.

The Test Planning Tool (TPT) had two primary goals. First, the TPT should allow an optical test planner to formulate a test without being completely knowledgeable about all flight and ground system operations. Second, the TPT should provide for the normal deviations and modifications inherent in optical testing while minimizing the additional overhead required to maintain strict flight hardware handling, quality assurance, test traceability, and configuration control.

The TPT software acted as a consolidated knowledge repository for optical test, optical hardware, and optical test operations. Any data relevant to possible optical test configurations or transitions between configurations was incorporated into the TPT. This information was integrated into the TPT in various ways. Constraints and limitations, for example, were often directly coded into checks run against an OTP that could produce errors or warnings. Individual mechanism controls could enforce limitations interactively as a user selects a configuration. The TPT also included a fully integrated content-sensitive help system that could be called up from any dialog window.

The process of incorporating this knowledge into the TPT improved communication and understanding between all engineers, analysts, and scientists as well as contractors, civil servants, and management involved in the optical test effort. We relied on communication from external stakeholders, flight operations experts, and sub-system leads to capture concerns, limitations, constraints, and best practices into the TPT. Once we incorporated that knowledge into the TPT, we made sure to walk the subject expert through how that information was captured in the TPT to ensure that it was implemented correctly. Additionally, special regression tests were designed to stress test the logic captured in the test planning tool.

This knowledge gathering, incorporation, and testing process helped form trust and understanding with all those involved in the optical test campaign. The improved communication, mutual understanding, and trust that knowledge has been accurately incorporated into the TPT helped minimize the number of critical personnel required to produce draft tests; we could avoid holding large meetings to ensure minor changes to tests were acceptable. The test planner could be a single point of contact for the data analyst or stakeholder for a particular test. This gives the test planner the best chance of designing an optimally efficient test that fulfills the data quality requirements while minimizing the risk of violating any constraints or limitations. In the pre-test planning stage, the final proposed test would then be reviewed by the larger optical test planning committees for approval. During the cryo-vac testing, test additions and modifications would be made by those who had experience developing OTPs

This methodology expedited the development of tests in preparation for the cryo-vac test, and it was absolutely critical when tests had to be modified or added during the cryo-vac test. However, this never stripped sub-system leads from reviewing or bringing up issues with tests before execution; however, it reduces any likelihood that there would be such issues.

Although the TPT is meant to be a compilation of as much relevant knowledge as possible, the TPT is not intended to remove experts from the test process. However, incorporating their expertise at the ground level, within the TPT when possible, avoids errors during test construction and minimizes the potential issues that could be discovered when the OTP is under review for approval.

\section{Core Architecture of the Test Planning Tool}

TPT software philosophy emphasized modularity and adaptability from the outset. This philosophy and subsequent software architecture was primarily motivated by changes to instrument hardware and control software that had previously occurred and continued to occur during the development of the TPT. The underlying structure of the ISIM Optical Test Planning Tool most closely resembles a state machine that is meshed with a simplified optical propagation model. Every potentially configurable item in an activity is represented by a state object. Configurable and nonconfigurable hardware elements that can alter the light travelling from source to detector are represented by a sub-class of the state object which we call a "node." State objects and nodes all have a special subset of properties that fully define the configuration of the item it represents. We call this subset of properties "the state." All state objects and nodes have three core methods: a method to retrieve the current state (getState ()), a method that attempts to apply a new state

\footnotetext{
${ }^{2}$ There is some ambiguity as to what the acronym "OTP" stands for. Optical Test Plan, Optical Test Procedure, and Optical Test "Proc" (i.e. a scripted procedure) are some of the variations.
} 
(setState()), and a method that returns information on the last transition of the object into its current state (getDelta()).

All the component objects are organized into a hierarchical structure. For example, each of the many OSIM sources is represented by an object that contains its current state; however, all those sources are contained within an object that represents OSIM's Source Delivery Module (SDM). That object has its own getState, setState, and getDelta methods. The SDM provides an amazing array of source wavelengths, attenuations, source-specific settings, and pulse control settings that can produce a near infinite number of illumination configurations. However, even though the SDM provides immense flexibility, there are still a significant number of prohibited configuration combinations. The SDM object, which contains all of the source objects and all additional SDM configuration information, can ensure that the aggregate SDM configuration is valid. In this example, when one or more sources are re-configured, the new composite SDM state is input into the setState() method. The SDM's setState() method performs global SDM configuration checks; if those checks pass, then each SDM component objects' setState() methods are called. If the composite logic in the SDM setState() fails or any of the component setState() methods fail, then the configuration is not valid and the SDM object's setState() method will fail. Conversely the SDM object's getState() method calls all the component objects' getState() method and compiles all of the state information for the entire composite SDM object.

The aggregated state structure from the object hierarchy defines a test activity. In Figure 3-2 the state objects and nodes whose combined states define an entire activity are highlighted in yellow. An "OTP" is an ordered set of activities that are represented by these state structures. The TPT evaluates a test by applying the aggregated configuration state for each activity in the designated order. Information generated by each state transition is used to check for errors, produce warnings, and generate estimates of execution time, limited life mechanism usage, data volume, and maximum data rate.

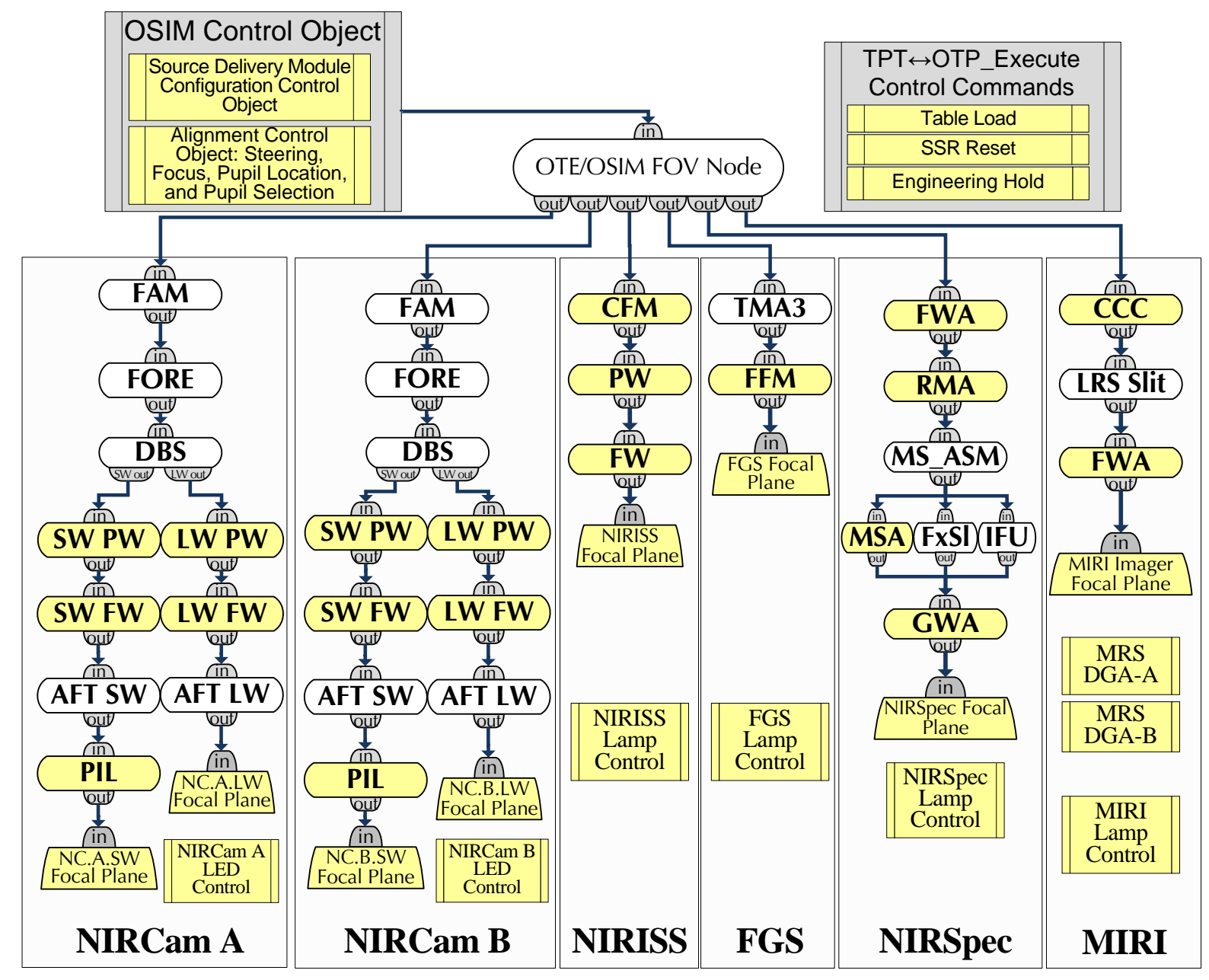

Figure 3-2 ISIM-OSIM Configurable Objects and the TPT Node Diagram 
Figure 3-2 also shows the optical node connectivity. When an OSIM source is illuminated, an "exposure path" object is created and then propagated from the OSIM simulated OTE focal surface through the chain of connected nodes until it terminates at the focal surface node. When an exposure path propagates through a node, the node's optical properties can be attached to the exposure path based on its current configuration. Additionally, certain nodes can perform operations on the exposure path itself. For example, a node may set the exposure path's next node as a function of the object's field coordinate. Also, a node may split an exposure path (e.g. at a beam-splitter) and attach different optical properties (e.g. transmission curves) to each path. This process is shown in more detail in Figure 3-3.

\section{Exposure Path Flow used for Image Simulation and Exposure Optimization}

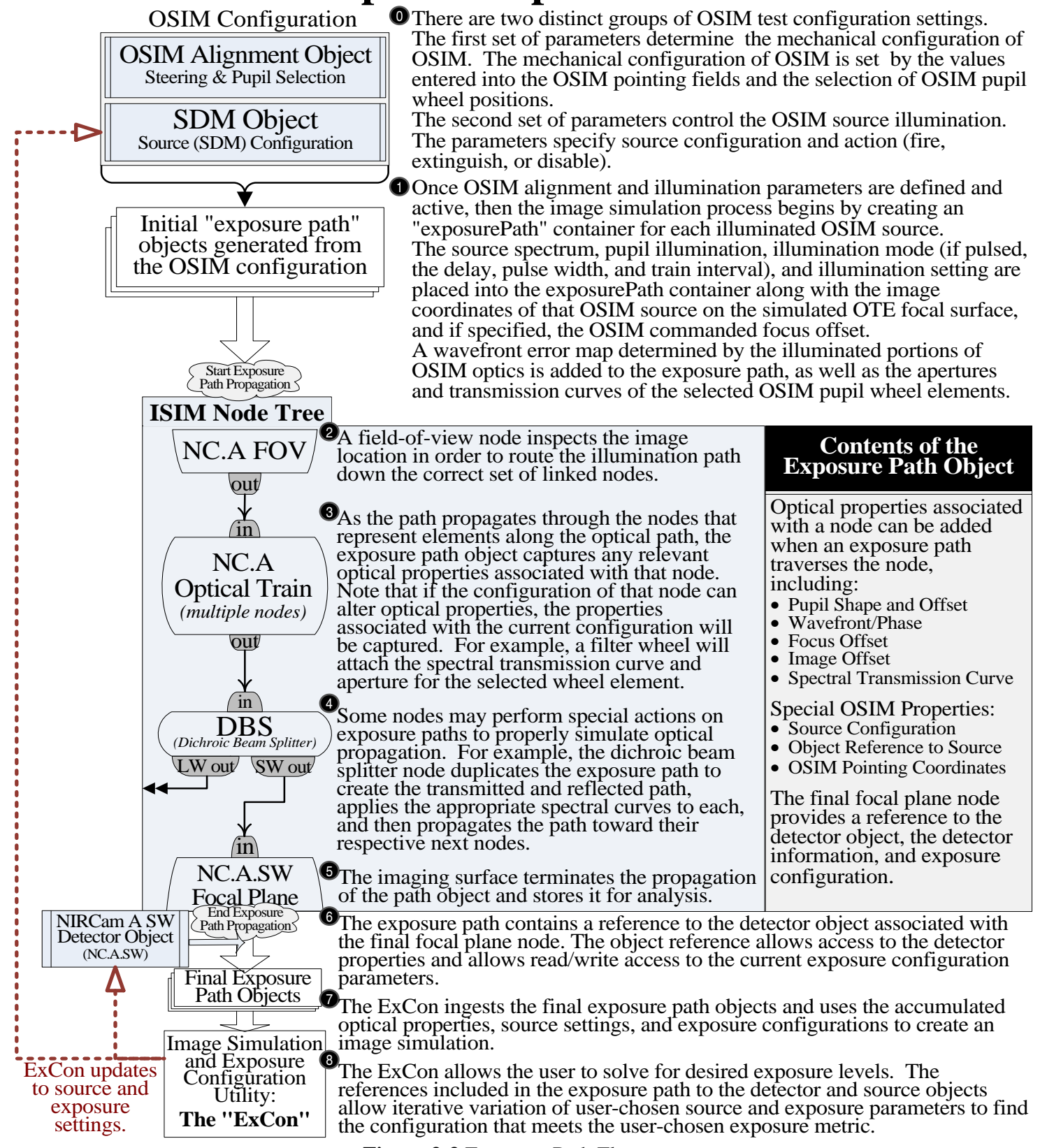

Figure 3-3 Exposure Path Flow 


\subsection{Overview of the Test Planning Tool Interface and Capabilities The ISIM/OSIM Timeline Tool}

The ISIM/OSIM Timeline Tool has three major sections:

A - The Test Tree B - The Test Table

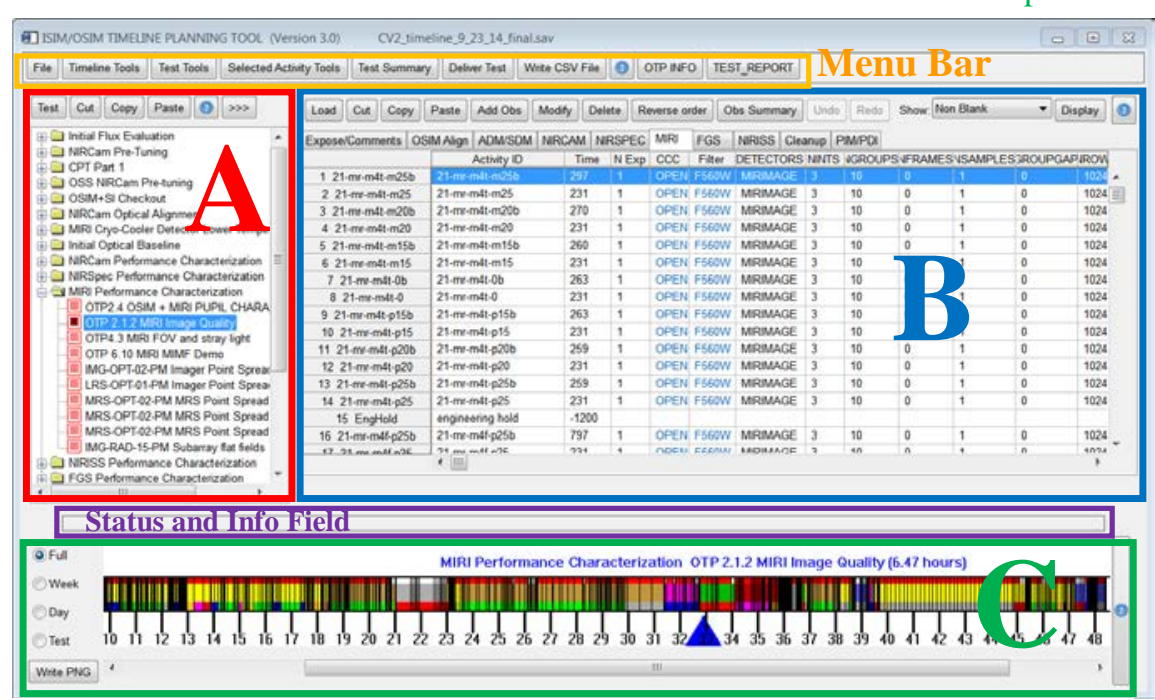

Figure 3-4 The Test Planning Tool Timeline Interface
C- Graphical Illustration of the Test Timeline

Additionally there is a menu bar and a status and informational field.

Status and Info Field:

Immediately above the graphical timeline picture is an informational field used to display progress or error messages for individual activities.

Menu Bar:

At the top of the tool is a menu bar that provides access to many key TPT functions:

The File button allows the user to save, restore, or clear the timeline, write csv files, and quit. The

Timeline Tools menu contains special purpose tools to apply to the full timeline. The Test Tools menu button contains special purpose tools to

apply to specific tests. The Selected Activity Tools contains special purpose tools for individual highlighted activities. The Test Summary evaluates the whole OTP sequence and produces the OTP summary information along with any errors or warnings. The Deliver Test button generates the final delivery package for the test. The Write CSV File button generates the review package for the test. The OTP Info button launches the OTP Info GUI. The Test Report button launches the Test Report GUI, from which the test planner's shift reports are generated.

\section{Timeline Tool - Test Tables}

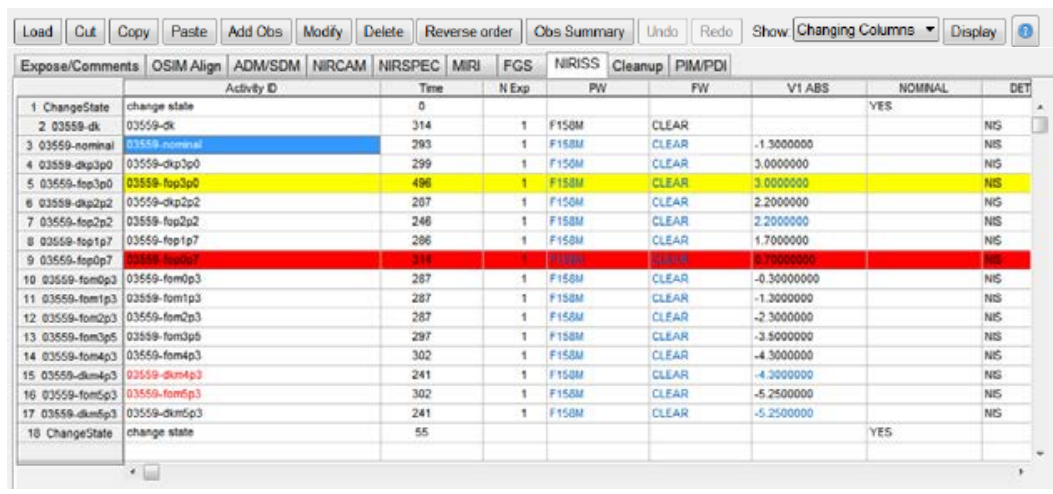

Figure 3-5 The Timeline Tool Test Table Section
The Test Table section of the Timeline Tool has three main parts:

- Menu bar (Load, Cut, Paste, etc.)

- Table Selection Tabs

- The selected portion of the Test Table The Table Selection Tabs control which portion of the Test Table to view. In the above graphic, NIRISS is selected. It shows 18 activities and table columns for NIRISS. To change to another instrument or portion of the table, select the appropriate tab above the table.

The background colors in the table indicate:

Blue - The currently selected activity or activities.

Yellow - The activity contains a warning.

Red - The activity generated an error condition (e.g. a constraint violation).

The errors and warnings are generated after running the Test Summary from the main menu. When an activity with a warning or error is selected, the details of the error will be displayed in the status and info field. Errors are also shown in the summary windows generated by the test summary and in the OTPInfo dialog. If any activity has an error (red background), the user will be unable to deliver the test.

When the activity ID shows up with red text (activities 15 and 16 in the above example), there is a question mark in one of the comment fields. This indicates an unresolved question concerning the activity. For example, the user may not know the correct current to use for an internal lamp. A test cannot be delivered when an unresolved question is present. 
The individual cells will be blank if the cell is not applicable to the current activity or the mechanism is flagged as not active. If the field is light blue, the field is active and has the current value. However, no command is needed since it has already been commanded in a previous activity.

Test Planning Tool Activity Configuration Interface

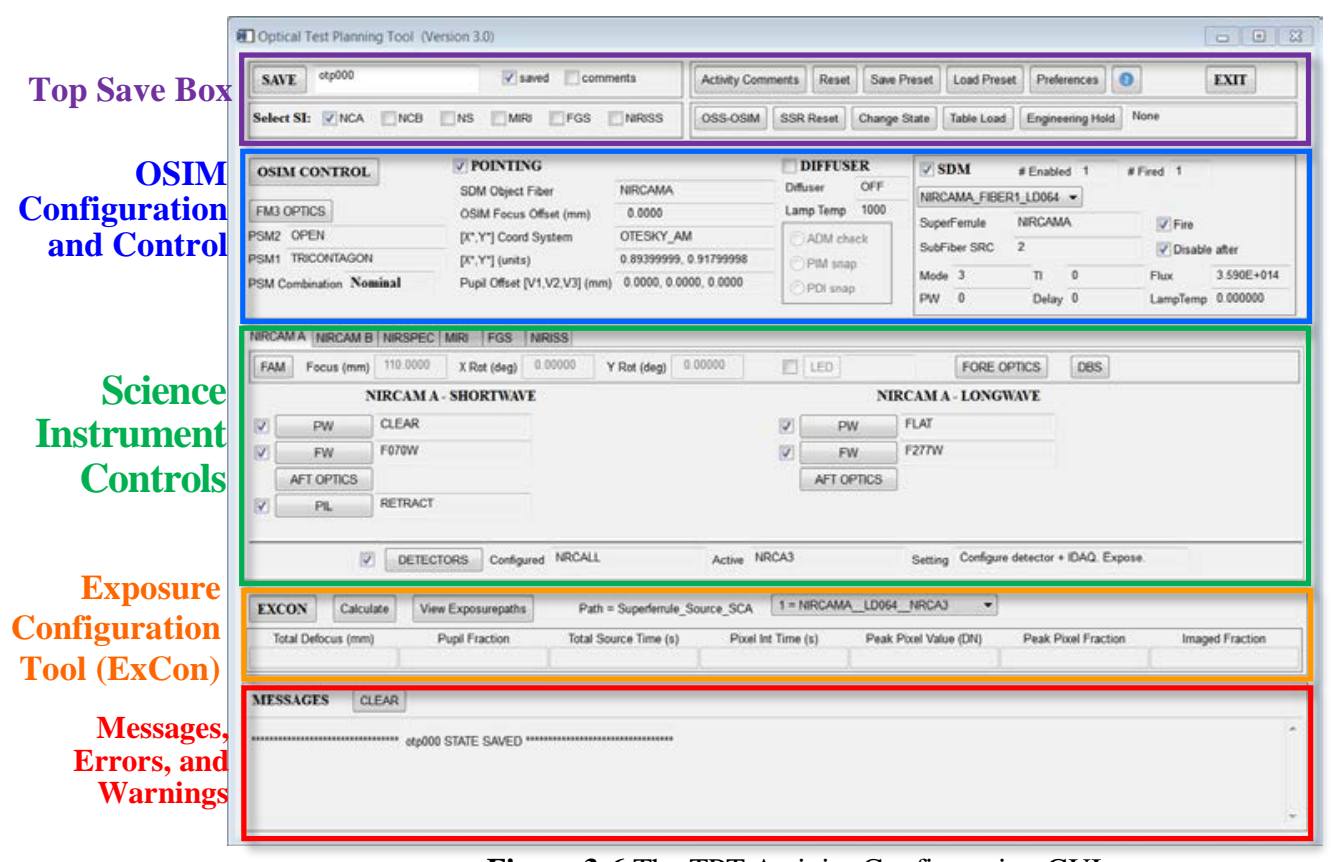

The Test Planning Tool Graphical User Interface (TPT GUI) is used to plan individual activities within an optical test. The TPT GUI can be operated as a standalone widget or within the Timeline Planning Tool. After each activity is completed, the $S A V E$ button will send the activity to the Timeline Tool, where it will be displayed on a single line of the table.

The TPT GUI is divided into 5 main sections shown in

Figure 3-6 The TPT Activity Configuration GUI Figure 3-6.

The top save box has a field to enter a 14 character activity label that will become part of the observation ID for any exposures taken during this activity. Below that field, there are SI selection boxes that allow a user to activate or deactivate all components in an instrument. This could also be done manually in each science instrument control tab. The OSS-OSIM button is only used for tests where an OTP is being used in concert with an OSS script. The SSR Reset button makes the entire activity a request to flush any unsent data in the SSR. The table load button allows a user to choose from a pre-defined list of software table loads. The engineering hold button provides the ability to turn the activity into a timed wait, or the engineering hold can pause OTP execution after suppling a message to the test operator indicating what action must be performed or completed before the Test Operator can resume OTP execution.

\section{OSIM Control Interface}

The OSIM Control Graphical User Interface (OSIM GUI) gives the user a way to select both how OSIM is pointed and what OSIM source or sources are in use. In addition, separate GUIs can be used to set up an ADM Check activity, a PIM Snap image, or a PDI Snap image. Figure 3-7 shows the main body of the OSIM GUI, launched by pressing the OSIM CONTROL button on the TPT GUI.

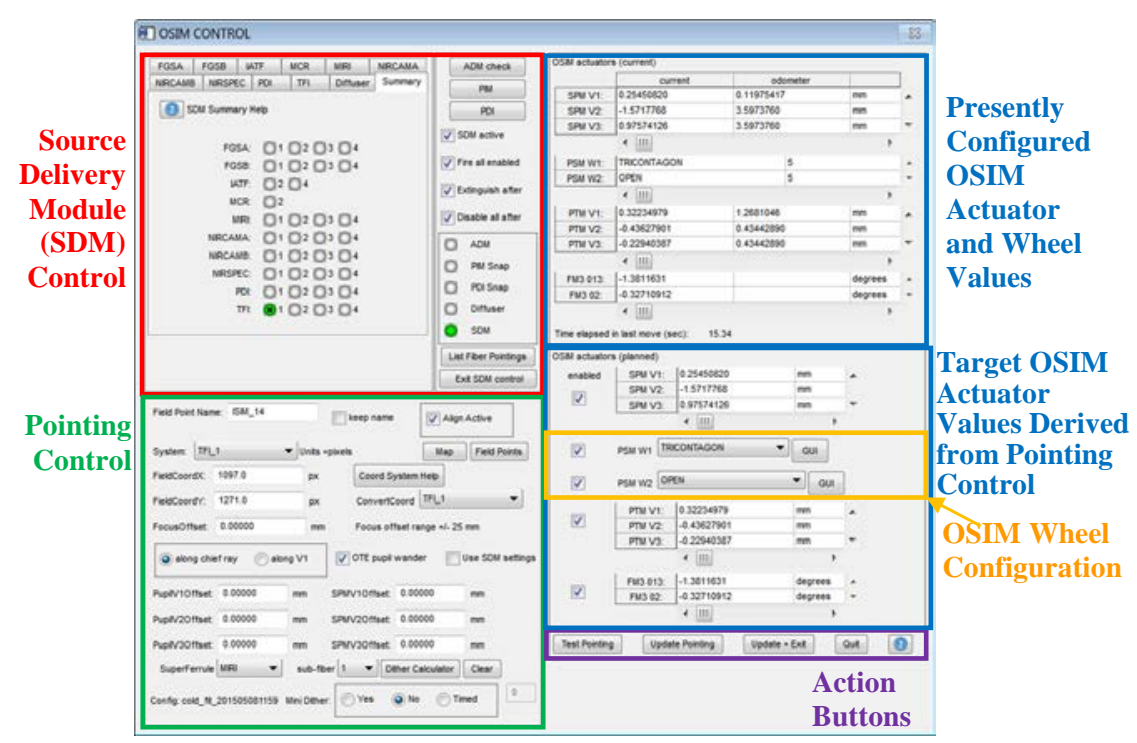

Figure 3-7 OSIM Control Interface 


\section{SI Configuration Interface Example: NIRSpec}

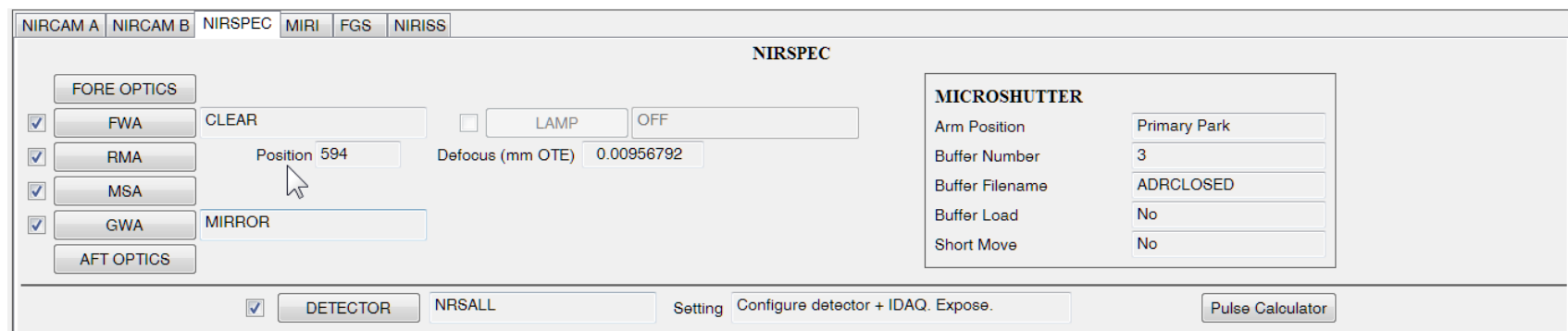

Figure 3-8 NIRSpec Configuration Tab

In Figure 3-6 The TPT Activity Configuration GUI has an area for Science Instrument (SI) configuration. Each instrument has a tab where configuration information is displayed and the configuration GUIs for the various components are accessible.

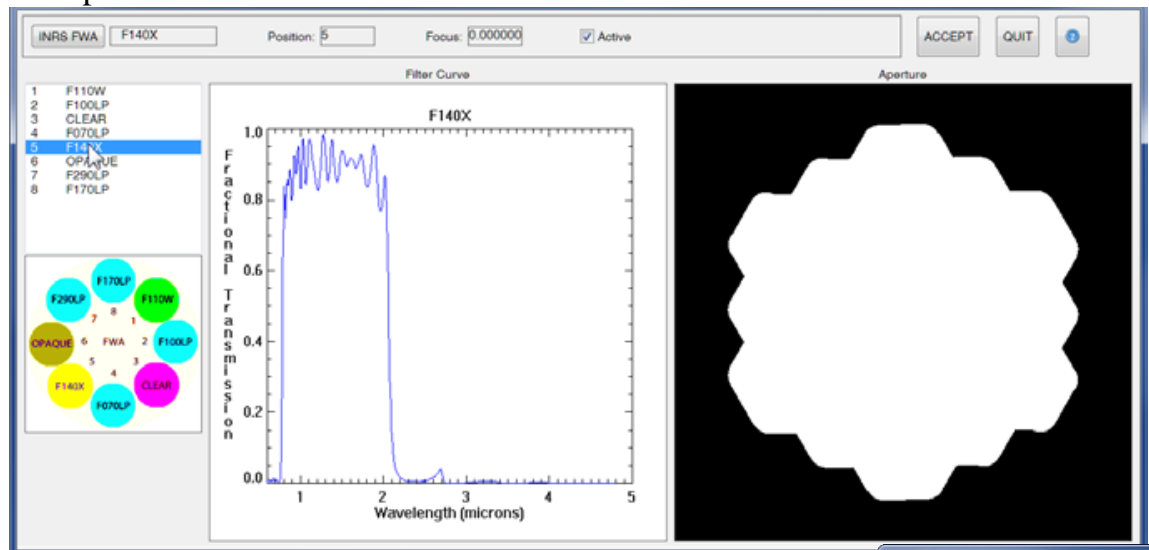

NIRSpec is used as an example of one of the SI tabs (Figure 3-8). There are a series of buttons down the left hand side that correspond to different SI elements. To the right of those buttons are fields that indicate the current configuration of that element. The microshutter array (MSA) configuration information is located on the right of this tab.

The buttons on the NIRSpec Configuration Tab call up graphical interfaces that either provide

Figure 3-9 NIRSpec FWA Interface

information or enable the user to configure that element. The top button, 'FORE OPTICS,' displays the transmission curve associated with the NIRSpec fore-optics.

The Filter Wheel Assembly (FWA) button calls up an interface (Figure 3-9) that provides transmission curves for each element, the aperture shape (if relevant), and the location of all the elements in the wheel. There are many filter/pupil wheels in ISIM, and their configuration interfaces are essentially uniform.

The Refocusing Mechanism Assembly (RMA) interface (Figure 3-10) provides several means of operating the RMA. The logic and rules regarding different move types and sizes is built into this interface. Entering the desired move and then clicking the RUN button checks the validity of the move request. The RMA control interface also maintains the conversions from the various focus mechanism coordinates.

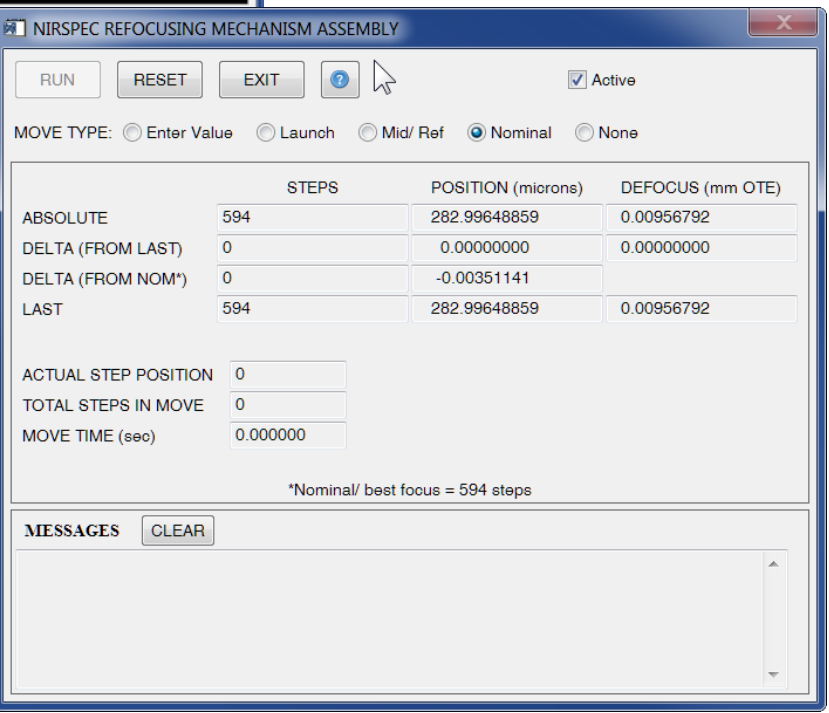

Figure 3-10 RMA Control Interface

The Microshutter Array (MSA) control interface (Figure 3-11) allows microshutter patterns to be loaded into the MSA buffer, enables microshutter patterns to be programed onto the array by actuating the magnetic armature (mag arm), and also allows the mag arm to move to between a set of locations without altering the shutter configuration. The display shows the chosen microshutter pattern and can overlay the known bad shutter mask to evaluate its impact. 


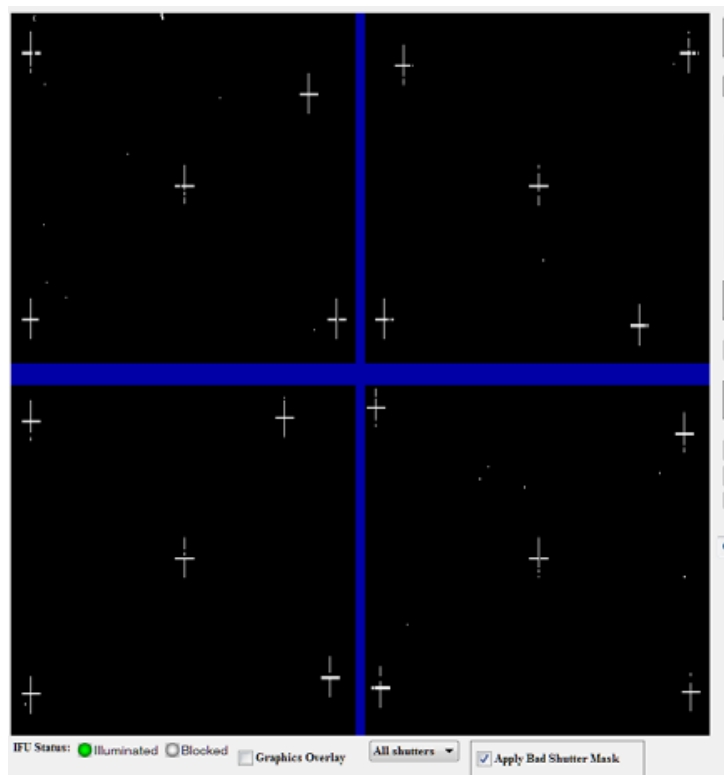

Figure 3-11 MSA Control Interface

\section{Exposure Configuration Utility, the "ExCon"}

The ExCon is the nexus of exposure configuration. The ExCon produces image simulations based on each exposure path object. That is, an object that started from an illuminated OSIM source and traversed the TPT node tree until it reached a focal plane object.

The ExCon produces an image simulation with the OSIM source configuration, the OSIM pointing information (including defocus), the aggregated transmission curves, apertures, and wavefront error maps, any focus offsets, and various shifts of image or pupil location correlated with the position of optomechanical mechanisms.

The process of configuring an exposure often requires adjustments to the illumination configuration as well as the exposure configuration. The ExCon provides access to both. So within the ExCon, a user can attempt to optimize an illumination and exposure setting to achieve the desired DN in the exposure.

The usefulness of this tool relies on how well the OSIM sources are characterized as a function of source configuration. Initially, the source models were based on results from OSIM commissioning; however, during the ISIM testing, some of the sources models were not accurate enough to produce useful results, and therefore we could not predictably determine the source and exposure configurations that would produce a well exposed image. Since the ExCon is an empirical tool to set source and exposure configurations, re-calibrating the source models could be done based on the set of data taken with that source during the ISIM CV test. An example of the analysis and process associated with an update to the EXCON source models is described by Reichard et al. (2016) ${ }^{11}$.
The detector configuration GUI (Figure 3-12) is modular in a manner similar to the filter wheel GUI, although in this case, instruments have a wider variety of capabilities and constraints. There are many detector configuration parameters; however, once a general detector readout mode is established, only one or two parameters are tuned for a given exposure. This interface, though, does allow the detector to be configured in any allowable state.

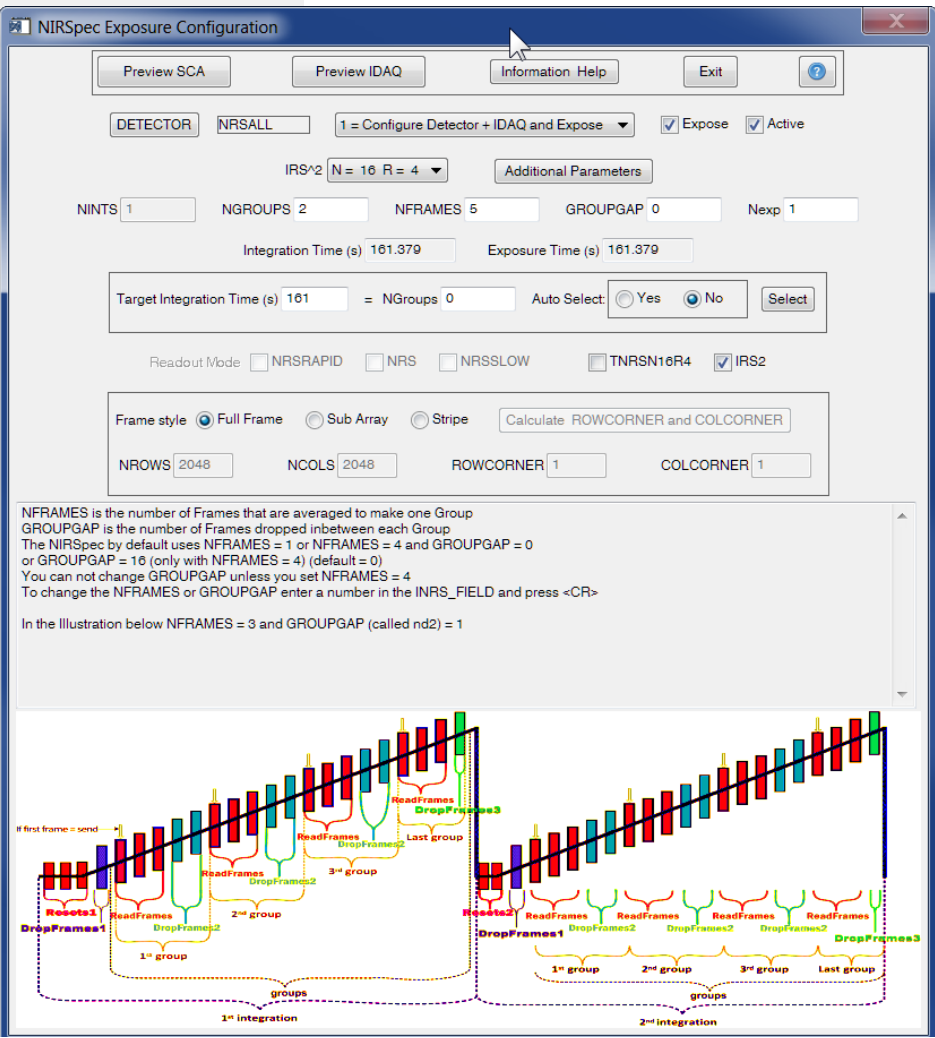

Figure 3-12 NIRSpec Exposure Configuration Interface
Mist Butfer / Mased (Lathes)

$9 / 69$ _SPCB $F$

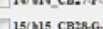

T16/16_CBs:P.PB

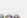

\author{
.
}




\section{The Exposure Configuration Utility, the "ExCon"}

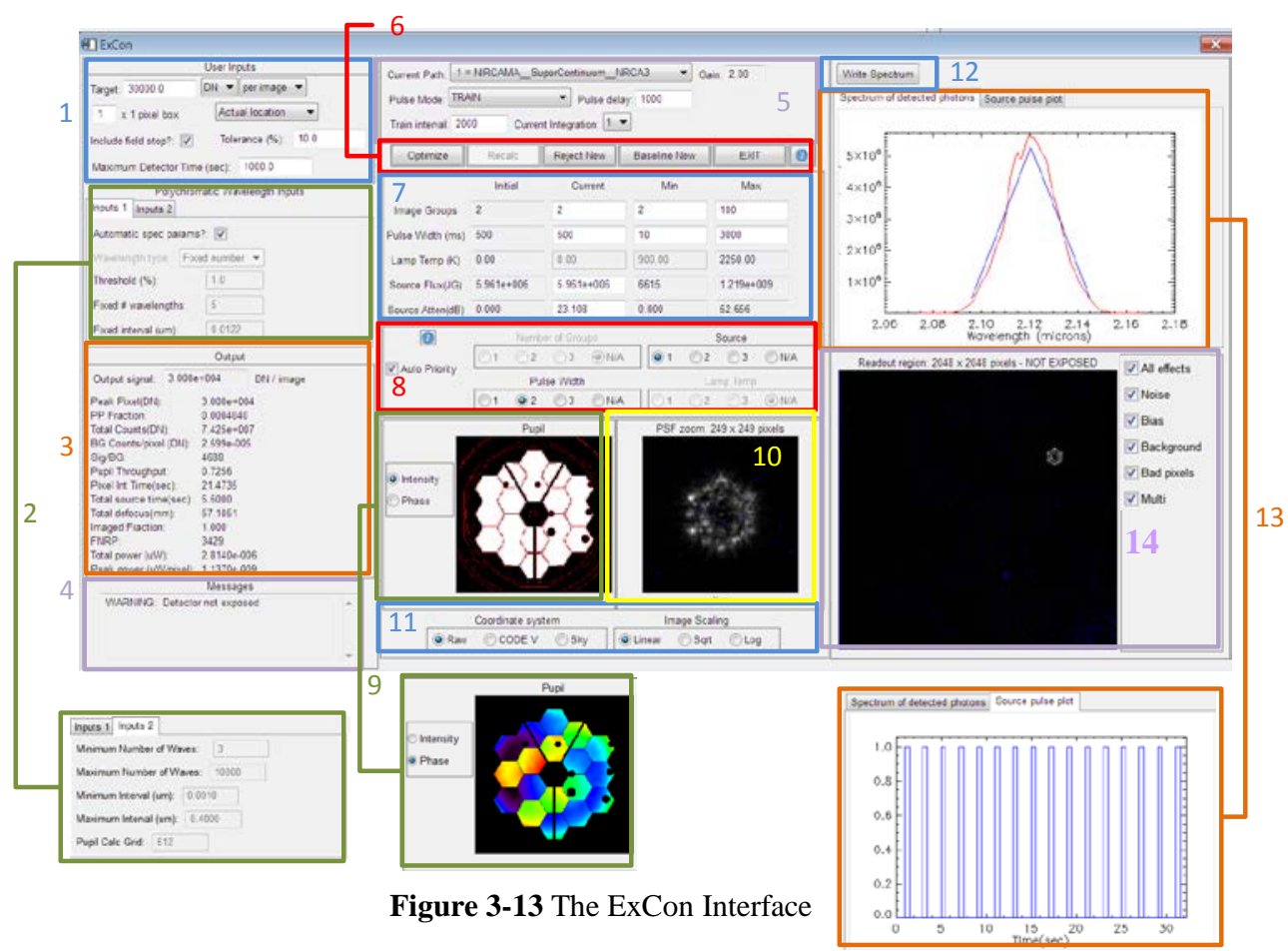

1. User inputs - This is the area where the user defines the target signal value and units for optimization.

2. Polychromatic Wavelength Inputs -Par7ameters that can be adjusted and determine how the code breaks down the spectrum of detected photons into a small set of wavelengths and weightings to calculate the polychromatic PSF.

3. Output - This area contains numerical outputs of the simulated image computation.

4. Messages - This area presents any errors or warnings resulting from the computation.

5. Path and pulse information - This area allows you to select the path number of interest and displays useful information about the SCA and source. It also has fields for configuring pulse parameters.

6. Action buttons - This area provides buttons to initiate calculations and perform optimization operations.

7. Parameter buttons - The fields in this area show the initial, current, minimum and maximum values for parameters for which optimization is allowed.

8. Priority buttons - These buttons allow the user to specify which parameters, and in which order, will be used for signal optimization. If the "Auto Priority" button is selected, then the priorities will automatically be set to a default sequence.

9. Pupil display - If "Intensity" is selected, the user will see an image of the composite pupil of the system, including all pupil planes in the optical path, which can include both OSIM and SI pupils. Red lines indicate the edges of the pupil apertures throughput the path. If "phase" is selected, the ExCon will display an OSIM phase map as derived from the OSIM CODE V model, excluding tilt and defocus. This phase is included in the computation.

10. PSF zoom - This shows a subregion of the full image containing most of the signal.

11. Image display options - The output images can be displayed either in "Raw" coordinates, "CODE V" coordinates as exist in the working optical models, or "Sky" coordinates as projected on the sky.

12. Write spectrum - This menu allows you to save the source spectrum.

13. Source plots - The two tabs here contain representations of the source.

a. "Spectrum of detected photons" shows in red the final spectral distribution propagated through the system, including the original source spectrum, the throughput of the various SI optics including selectable filters, and the detector quantum efficiency. The blue line shows the spectrum after being rebinned into a smaller set of wavelengths for computing the polychromatic PSF, using the parameters as described in area 2.

b. "Source pulse plot" shows the source temporal pulse profile over the course of the integration, with a value of 1 indicating when the shutter is open and 0 indicating closed.

14. Full image - This area shows an image representation of the image region as configured. If the image is full frame, this will show the entire SCA. If the image is a subarray, only the subarray region will be shown. The heading of the window indicates the size of the image in pixels and whether or not the image is exposed. The boxes to the right offer calculation options for the simulated image. None of these options affect the flux computations, only the appearance of the simulated images. 


\section{OTP Info GUI}

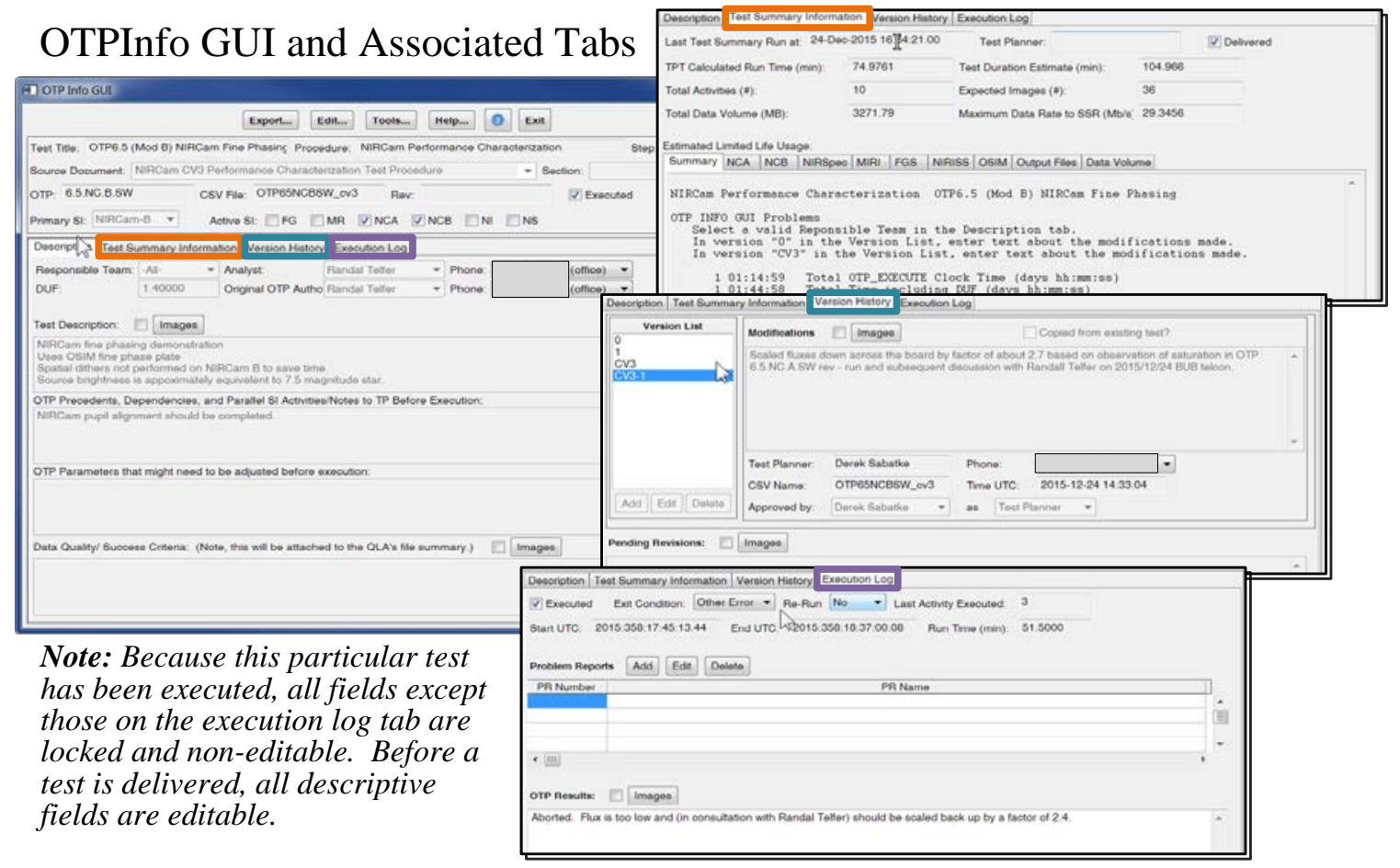

Figure 3-14 The OTPInfo GUI

The Description tab contains specific overview information about the test. Of particular importance are the Test Description and Data Quality sections. The Test Description field is meant to record the intent of the test and the rationale for how the OTP is designed. The Data Quality should note any unique qualities or criteria that need to be checked. This field is written into the file summary spreadsheet that the quick-look analyst fills out. Images may be attached to most of these fields.

If a test summary has been run in the Timeline Tool, the Test Summary Information tab will contain a list of any errors and warnings, the estimated execution timing, limited life usage for each instrument, a list of output files, the total data volume, and the maximum data transfer rate.

The Version History tab contains the modification history of the OTP as well as any previously executed tests that the current test is derived from. The Version List at the left shows the names of existing versions of the test. The information about each version/revision includes a description of what was modified, whether the version was copied from an existing test in the timeline, the Test Planner who authored the version, the Test Planner's phone numbers, the OTP CSV filename, the time the version was created, and the approval status, including the approver if the version was approved.

The Execution Log Tab will display the status of the test. If it has been run, the Executed box will be checked. The test planner inputs the exit condition and if a re-run is required. The Timeline Tool will fill in the Start and End UTC as well as the Run Time. If there are any Problem Reports associated with the execution or results of this OTP, the PR reference can be recorded here. A description of the results of the OTP execution description is entered by the test planner here and is included in the test summary report.

\subsection{DHAS and Quick-look Tools}

The quick-look operator uses the QUICKLOOK_CONTROL_GUI (Figure 3-15) to monitor data as received. This routine monitors the data catalogs that are updated by the ISIM DHAS. As new data are received, the routine will display information about the dataset in a table on the quick-look control console.

The Quick-Look Analyst (QLA) will determine if the incoming images meet the appropriate data quality criteria and will perform basic analysis tasks called for in the optical test procedures. A package of integrated image analysis routines, known as the 'Quick-Look Tools," (Figure 3-16) can be launched from the catalog monitoring software (Figure 3-15). 


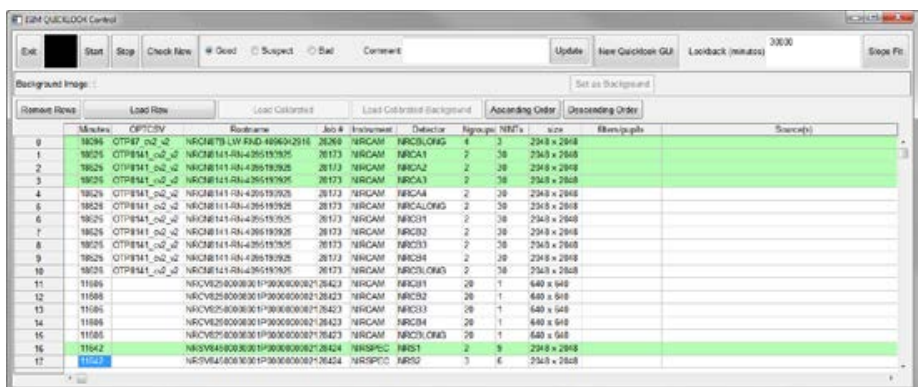

Figure 3-15 Catalog monitoring \& Quicklook Control GUI

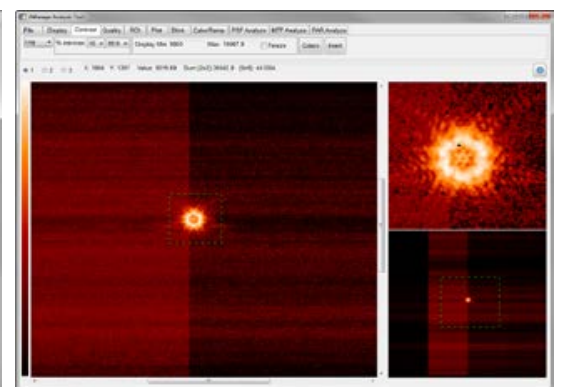

Figure 3-16 Quicklook Analysis Tool

Additionally, the ISIM DHAS keeps a catalog of the all the FITS keyword headers it has processed. This catalog can be searched with any set of criteria found in the FITS keyword headers. The search results are displayed in a configurable spreadsheet. If the software is running on a machine that has access to all of the ISIM Cryo-Vac data, then the data can be directly loaded into the quick look analysis tool.

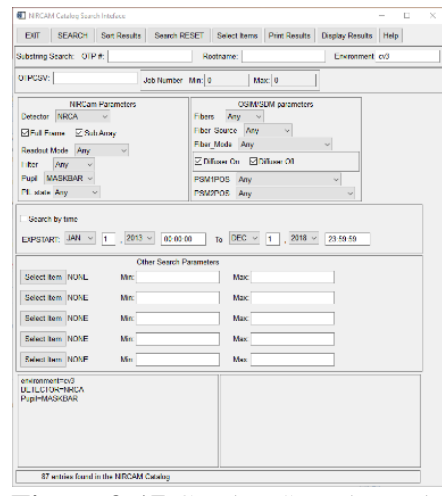

Figure 3-17 Catalog Search Tool

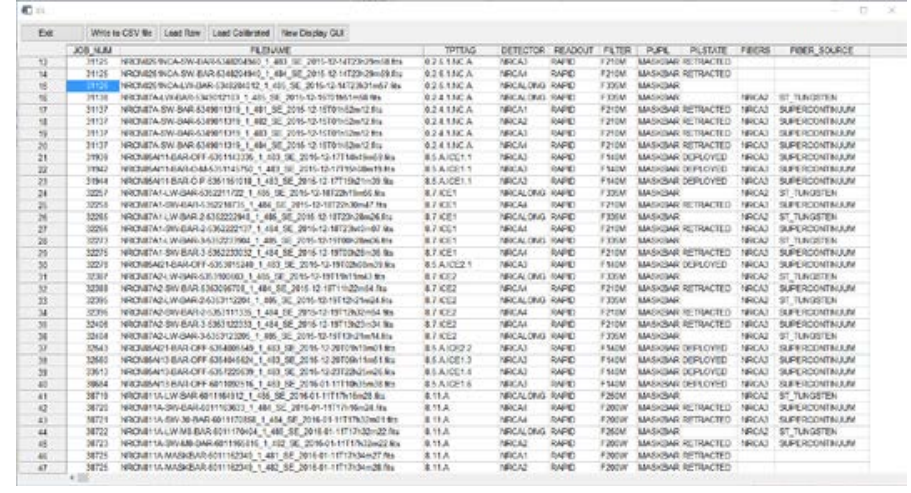

Figure 3-18 DHAS Catalog Search Results

General imaging data quality criteria are kept in the ISIM Optical Test Plan document, however, test and exposure specific data can be specified in the TPT. The TPT outputs a file summary spreadsheet that include these test and exposure specific quick look data criteria and is filled out by the Quick Look Analyst as shown in Figure 3-19.

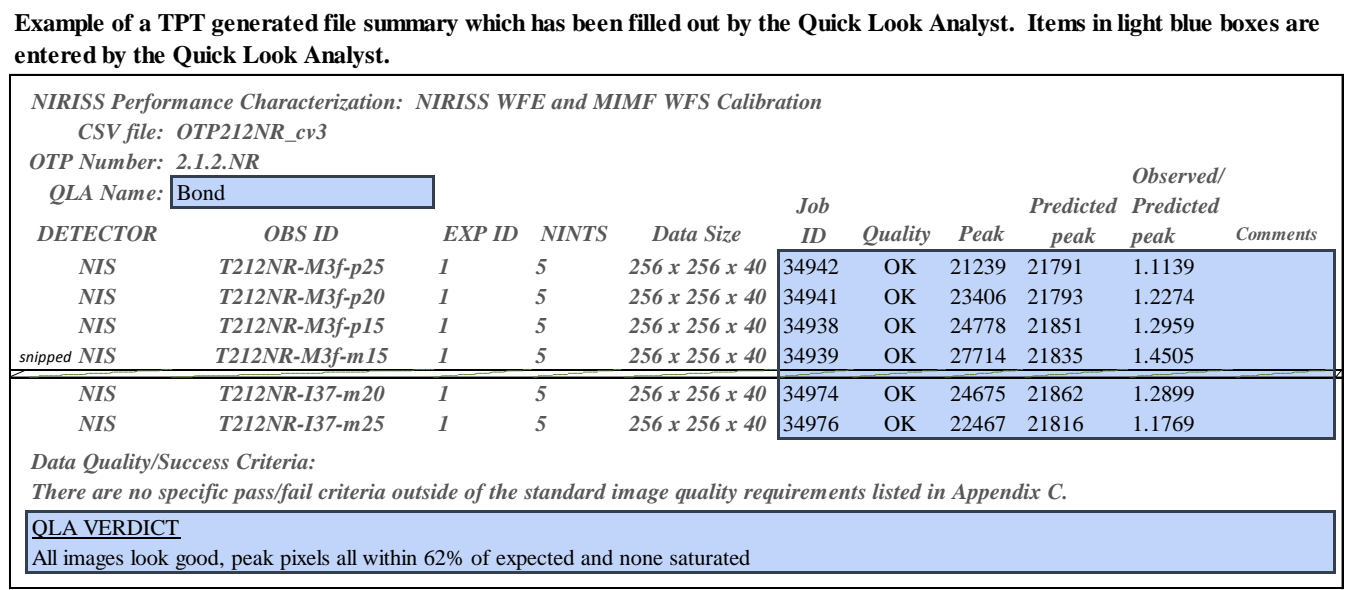

Figure 3-19 This is a 'File Summary' generated by the TPT during OTP delivery. Blue fields indicate the portions completed by the Quick-Look Analyst after the OTP was executed. 


\section{TEST AND PROCEDURE DEVELOPMENT}

Test and Procedure development could only start in earnest once the ISIM Optical Test Planning Tool (TPT) was functional and the performance and characterization requirements were translated to concrete test requirements, as discussed in Section 2. The primary products of this effort in our "Test Planning Tool paradigm" were the TPTgenerated test sequences (mostly referred to as "OTPs") and the test procedures. Although the OTPs and the test procedure documents are intrinsically linked, consolidating the granular test detail within the OTPs made the test procedures shorter, easier to review, and more focused on the test process.

A non-trivial step, and one that was surprisingly important step to complete early in this process, is to start identifying and organizing OTPs. Early in the test development process, considerable effort must be invested in cataloging the full set of discrete OTPs. This OTP list includes those required to provide the data needed by all the PCF as well as any prerequisite OTPs that provide any OGSE or flight hardware calibrations required to successfully execute and interpret subsequent OTPs. The nomenclature and organization of OTPs is also a critical part of providing meaningful labels to tests while also being precise and adaptable. If concerted effort is invested in constructing a good framework for identifying and organizing OTPs, the resultant nomenclature system can pay dividends from the very initial planning phases through post-test analysis.

\subsection{OTP Organization}

The goal of an Optical Test Proc (OTP) is to gather a purpose-oriented set of data. That dataset provides the necessary input into an ISIM-element PCF, an SI specific test, or in the most general sense, collects the data required for a specific analysis or to investigate a specific anomaly.

Tests focused on the ISIM-element requirements, discussed in Section 2 have PCF IDs associated with them. The OTP IDs reflect the PCF ID that the OTP test addresses. Some PCFs required multiple OTPs, the base PCF ID would be retained and an additional suffix would be added.

For example, pupil alignment requirements can be found in OPRG3. Because of the strong similarities involved in collecting and analyzing pupil image data from the various internal SI pupil alignment references (PARs), the associated measurements of OTE to SI pupil registration error were all bookkept under a single PCF. The corresponding OTPs then build off of that PCF ID in a hierarchical manner, so for example, PCF 3.1 requires measurements of instruments' internal pupil alignment references (PAR). So to uniquely identify the OTP that gathers the data for the NIRCam A channel, the assigned OTP ID is 3.1.NC.A.

The OTP ID hierarchy and nomenclature can help clarify some of the OTP variations between instruments as well as some of the subtle differences between similar OTPs done with the same instrument. A good example of this in the pupil-related tests is the FGS-Guider pupil alignment check. Although FGS-Guider provides two different imaging channels, the frontend of the instrument that includes the entrance pupil and pupil alignment reference are shared by both channels. Therefore, OTP IDs that perform imaging in single FGS-Guider channel have OTP IDs that indicate the FGS-Guider channel (e.g. OTP 2.4.FG.G1). However, because there is a single pupil alignment reference in FGS, the OTP ID is OTP 3.1.FG - signifying that this one test provides the necessary information for both guider channels. Also, OTPs meant to

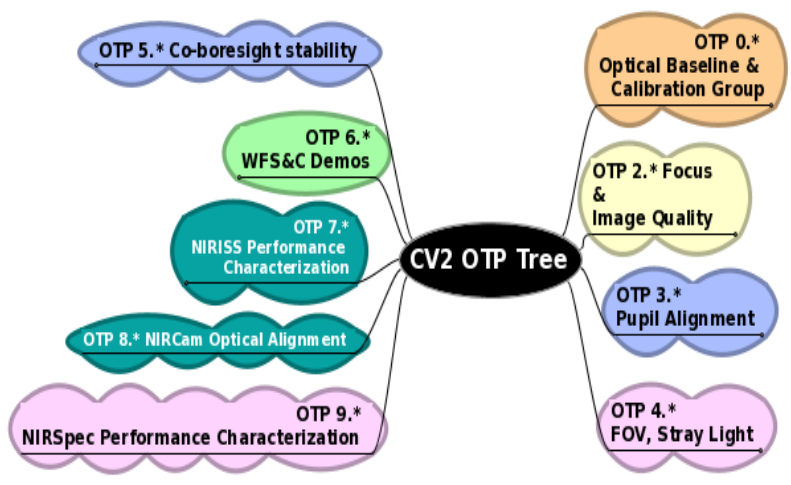

Figure 4-1 CV2 Top Level OTP Tree test perturbations in performance from environmental variations can be easily broken into OTP IDs such as OTP 0.4.2.NR.INIT and OTP 0.4.2.NR.FINAL.

Also, synergizing the PCF nomenclature with the hierarchical OTP nomenclature allowed for a sustainable organizational template to handle the constant churn of new OTPs being created, deleted, split, or modified while maintaining traceability back to the PCF where the data is utilized.

Once instrument teams and other subsystem test stakeholders began requesting additional tests, we expanded the OTP ID scheme beyond the six or seven PCF in order to add OTP ID prefixes numbers for instrument specific tests. Figure 4-1shows the top level OTP nomenclature and ID structure for ISIM Cryo-vac \#2. 


\subsection{Procedure Development}

Developing a standardized approach to writing optical test procedures that utilized the TPT/OTP paradigm made writing, reviewing, and executing the procedures easier and improved efficiency. However, the development of that standardization process took a considerable amount of trial and error.

Each ISIM Cryo-Vac test required more than a dozen separate procedures. One overarching procedure, the "Master" ISIM Cryo-Vac test procedure, called these sub-procedures as necessary. Many sub-procedures did not include optical testing, and those procedures did not follow the formulaic approach that we developed for sub-procedures dominated by optical testing.

The first realizable procedure standardization was a direct consequence of the TPT/OTP approach: the procedural steps involved in preparing, executing, and checking the results of an OTP were nearly identical for the many hundreds of OTPs that needed to be run. Furthermore, when an OTP redline or blackline insertion was necessary, the "fill in the blank" template developed for writing the initial procedure was available electronically and could be quickly edited to produce the procedural steps that needed to be inserted into the as-run procedure.

An additional adjustment made to OTP dominated tests was to minimize or eliminate text in the procedure that already existed in the TPT and associated OTP. By keeping the fine optical test operation details in the TPT and OTPs, the procedures became more focused and less cluttered.

Significant improvements to our optical test procedure template came from lessons learned from during our first ISIM cryo-vac test cycle. A primary and widely prevalent problem was that using a compact, rigid, and success-oriented procedure style became a mess as soon as blacklines were added and tests were re-ordered. The as-run procedures were impossible to interpret or sequence, to the point where there was confusion from shift to shift as to what had been done. Those earlier formats lacked the flexibility to handle the reality of testing.

However, we could not move to the other extreme. A list of tests followed by a free-form "fill in the blank" procedure to be filled out at run time would weaken the formal review process and make it difficult to ensure that special steps, checks, and gates were inserted where they were needed.

After consideration of these potential pitfalls and additional trial and error, the optical test procedure template evolved into the following basic format.

The procedure was divided into several major blocks. The very first block of the procedure contained steps to initialize, configure, or confirm that the ground system, OGSE, and flight hardware were prepared to start optical testing. Similarly, the last block contained any steps necessary to re-configure, capture logs, or perform any other clean-up activities so that the ground system, OGSE, and flight hardware were prepared to move onto the next procedure.

The blocks contained in the body of the procedure were either blocks of OTPs or a block of procedural steps that were required between blocks of OTPs.

OTPs that shared a purpose and had similar or identical entrance and exit criteria were grouped into blocks.

- The first section of the block, the "header" or "test list" section, contained directive instructions and information (purpose, entrance criteria) concerning that block of tests

- A table listing all of the nominally planned OTPs. NOTE: The OTP table in the head section also contained many blank rows to accommodate the OTP re-runs, modifications, and additions relevant to that section.

\begin{tabular}{|c|c|c|c|c|c|c|c|c|c|}
\hline \multirow{3}{*}{2.1.} & \multicolumn{9}{|c|}{ TIILE OF FIRST (or only) OIP BLOGK } \\
\hline & \multicolumn{9}{|c|}{ OTP Section Description, Contents, and Execution Details } \\
\hline & \multicolumn{9}{|c|}{$\begin{array}{l}\text { Description: } \\
\text { Entrance Criteria: } *\end{array}$} \\
\hline \multirow{9}{*}{2.2.} & \multicolumn{9}{|c|}{ Title of OTP Block: OTP Test Table } \\
\hline & $\begin{array}{l}\text { Procedure } \\
\text { Step \# }\end{array}$ & $\begin{array}{l}\text { Test Name and preferred order of } \\
\text { execution }\end{array}$ & $\begin{array}{l}\text { OTP filename(s) } \\
\text { (boselinetests listedfist) }\end{array}$ & Priority & $\begin{array}{l}\text { Source document, } \\
\text { Section \# }\end{array}$ & $\begin{array}{l}\text { Dependencies, } \\
\text { Restrictions }\end{array}$ & $\begin{array}{l}\text { Parallel } \\
\text { ops }\end{array}$ & $\begin{array}{l}\text { Approx. run } \\
\text { time (hours) }\end{array}$ & $\begin{array}{l}\text { Complete - } \\
\text { no more }\end{array}$ \\
\hline & 2.2 .1 & $\begin{array}{l}\text { OTP 2.4 - OSIM + NIRSpec Pupil } \\
\text { Characterization }\end{array}$ & OTP24NS_CV3.bt & $\begin{array}{l}\text { Req Ver- } \\
\text { Pre/Post Env }\end{array}$ & $\begin{array}{l}\text { ISIM-OSIM CryoVac \#3 } \\
\text { Optical Test Plan, } \\
\text { \$5.4.3.2.1 }\end{array}$ & $\begin{array}{l}\text { Run after best focus } \\
\text { set in ofisin+SI } \\
\text { checkout }\end{array}$ & None & 1.07 & $\square$ final rev? \\
\hline & 2.2 .2 & $\begin{array}{l}\text { OTP 2.1.2 - Wavefront Error and } \\
\text { MIMF WFS Calibration }\end{array}$ & OTP212NS_CV3.tot & $\begin{array}{l}\text { Req Ver- } \\
\text { Pre/Post Env }\end{array}$ & & & None & 3.09 & 口 final rev? \\
\hline & 2.2. $x$ & ... [remaining OTPs in block] & $\ldots$ & ... & $\ldots$ & $\ldots$ & $\ldots$ & $\ldots$ & $\square$ final rev? \\
\hline & 2.2.8 & $\begin{array}{l}\text { OTP } 4.3 \text { - SI Field of View and Stray Light } \\
\text { checks }\end{array}$ & OTP43NS_CV3.txt & $\begin{array}{l}\text { ReqVer- } \\
\text { Pre/Post Env }\end{array}$ & $\begin{array}{l}\text { ISIM-OSIM CryoVac \#3 } \\
\text { Optical Test Plan, §... }\end{array}$ & $\ldots$ & $\ldots$ & $\ldots$ & 口 final rev? \\
\hline & 2.2 .9 & $\begin{array}{l}\text { OTP } 2.4-0 S I M+N I R S p e c \text { Pupil } \\
\text { characterization, Rev A }\end{array}$ & Otrans_aratet & $\begin{array}{l}\text { Req Ver- } \\
\text { Pre/Post Env }\end{array}$ & & & & 0.6 & $\square$ final rev? \\
\hline & 2.2 .10 & $\begin{array}{l}\text { OTP } 2.4-O S I M+N I R \text { Spec Pupil } \\
\text { characterization, RevB }\end{array}$ & OTrans crusutat & $\begin{array}{l}\text { Req Ver- } \\
\text { Pre/Post Env }\end{array}$ & & & & 0.3 & 凶final rev? \\
\hline & $2.2 . x$ & $\begin{array}{l}\text {... [any additional OTPs inserted } \\
\text { by blacklíne] }\end{array}$ & & & & & & & $\square$ final rev? \\
\hline
\end{tabular}

Figure 4-2 OTP Execution Block Header 
- The second section contained all of the procedural steps to run the OTPs. When submitted for review, all of the procedural steps for the pre-planned OTPs were included.

o Blackline or Redline additions are inserted into the as-run procedure after the last pre-planned OTP in the test procedure

o If an OTP is re-run or added, the test conductor would add the OTP information to the as-run OTP test table as shown in blue text above.

\begin{tabular}{|c|c|c|c|c|c|c|c|}
\hline \begin{tabular}{|l|} 
Seq\#. \\
Step\# \\
\end{tabular} & \begin{tabular}{|c|} 
Resp \\
Initials \\
\end{tabular} & \begin{tabular}{|l|} 
Date/Time \\
Completed
\end{tabular} & Actionee & Action & Expected Result & \begin{tabular}{|l|} 
Expected \\
Duration \\
\end{tabular} & $\begin{array}{c}\mathrm{PR} \\
\# \\
\end{array}$ \\
\hline \multicolumn{8}{|c|}{ 4.2. OTP Execution Block: NIRSpec Performance Characterization } \\
\hline 4.2.1. & \multicolumn{7}{|c|}{ OTP 2.4 - OSIM + NIRSpec Pupil Characterization } \\
\hline 4.2.1.1. & & & TP & $\begin{array}{l}\text { Deliver OTP from TPT. } \\
\text { Commit output products to CV3OpticalOps SVN Repository. } \\
\text { Copy “.txt" version into the OTP_EXECUTE path. }\end{array}$ & OTP ready to execute & $4 \mathrm{~min}$ & \\
\hline 4.2.1.2. & & & TO & -Check OSIM and ISIM status to ensure system is ready to execute OTP & System ready & $2 \mathrm{~min}$ & \\
\hline 4.2.1.3. & & & TO & $\begin{array}{l}\text { Start OTP } 2.4 \text { - OSIM + NIRSpec Pupil Characterization } \\
\text { CECIL >START isim_otp_execute('OTP24NS_cv3.txt') } \\
\text { If the test suspends (manually or programmatically), do not completely abort out of the } \\
\text { ISIM_OTP_EXECUTE script. }\end{array}$ & Script completes without errors & ??? min & \\
\hline 4.2.1.4. & & & TO & 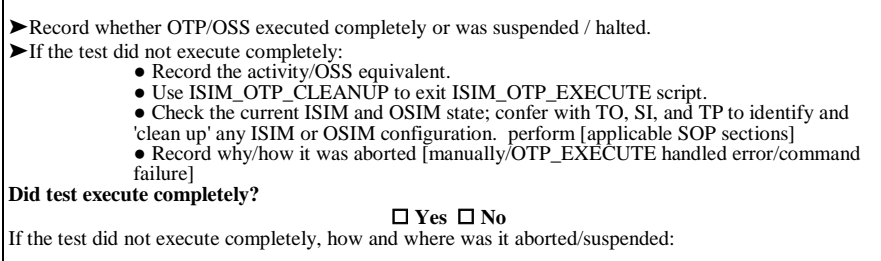 & $\begin{array}{l}\text { OTP_EXECUTE execution results } \\
\text { recorded. }\end{array}$ & $5 \min$ & \\
\hline 4.2.1.5. & & & $\begin{array}{c}\text { NS, } \\
\text { QLA, TP }\end{array}$ & $\begin{array}{l}\text { (QLA) Check data quality as described in JWST-PLAN-026322, ISIM-OSIM CryoVac \#3 Optical Test } \\
\text { Plan, Appendix C and/or as described in the TPT generated QLA log. } \\
\text { (QLA, NS) Check data quality and ensure there are no anomalous artifacts in the collected images. } \\
\text { (TC) Confer with NS, QLA, and TP and record the following: } \\
\text { Were all the data necessary collected? } \\
\text { Were the data quality criteria satisfied? } \\
\text { Yes } \square \text { No } \\
\text { No }\end{array}$ & \begin{tabular}{|l|} 
Either (1) QLA and NS confirm data \\
quality meets expectations or (2) log \\
observations that fail data quality \\
criteria and advise TP of changes \\
-If revision or re-run is needed, (TP) \\
delivers a revised state table
\end{tabular} & $5 \mathrm{~min}$ & \\
\hline 4.2.1.6. & & & & $\begin{array}{ll}\text { If all the required data has been collected and meets the data quality criteria, record this OTP run as complete. } \\
\text { If additional data needs to be collected: } \\
1 . \\
\text { (TP) Draft a new state table and increment the version letter. } \\
\text { 3. } & \text { (...) Have state table approved [ } \\
4 . & \text { Fill out a new OTP execution procedure template, } \\
\text { Insert template and [?] add the revision number to the Seq\#.Step\# (e.g. "2.2.a") }\end{array}$ & $\begin{array}{l}\text { All desired data associated with this } \\
\text { OTP has been collected, or a new } \\
\text { state table has been drafted to acquire } \\
\text { remaining data. }\end{array}$ & $5 \mathrm{~min}$ & \\
\hline 4.2.2. & \multicolumn{7}{|c|}{ OTP 2.1.2 - Wavefront Error and MIMF WFS Calibration } \\
\hline 4.2.2.1. & $\ldots$ & $\ldots$ & $\ldots$ & $\ldots$ & $\ldots$ & $\ldots$ & \\
\hline
\end{tabular}

Figure 4-3 Excerpt of OTP Execution Block

- The last section, the exit section, of each OTP block contained at least the basic exit criteria for that block.

\begin{tabular}{|c|c|c|c|c|c|c|c|}
\hline \begin{tabular}{|l|} 
Seq\#. \\
Step\#
\end{tabular} & $\begin{array}{l}\text { Resp } \\
\text { Initials }\end{array}$ & \begin{tabular}{l|} 
Date/Time \\
Completed
\end{tabular} & Actionee & Action & $\begin{array}{l}\text { Expected } \\
\text { Result }\end{array}$ & \begin{tabular}{|l|} 
Expected \\
Duration
\end{tabular} & PR\# \\
\hline 6.3. & \multicolumn{7}{|c|}{ Exit Criteria for NIRSpec Performance Characterization OTP/OSS Test Block } \\
\hline 6.3.1. & & & TC & $\begin{array}{l}\text { Have all the OTPs associated with this section (listed in 2.1.2) been executed? } \\
\text { Have all revised / added OTP/OSS tests in this section been logged and recorded? } \\
\text { Can testing proceed to the next section? } \square \text { Yes } \square \text { No } \\
\text { Is this section complete (i.e. have all exit criteria been met and test objectives for this section been met)? } \\
\text { Yes } \square \text { No }\end{array}$ & $\begin{array}{c}\text { Section } \\
\text { objectives } \\
\text { met. }\end{array}$ & $\mathbf{X}$ hrs & \\
\hline
\end{tabular}

Figure 4-4 OTP Execution Block Exit Criteria

o This could be as simple as checking that all the data designed to be collected in the nominal list of OTPs has been collected, or it could indicate that a more involved analysis and or re-calibration must be completed before moving on.

o It also could contain instructions on what testing could proceed if all exit criteria were not met.

$0 \quad$ E.g. If all the data from one instrument was collected, then the exit block of that OTP section could indicate that the OTPs for that instrument in the next section could be executed before all the exit criteria were met for that section.

- Any clean-up activities for that block were also indicated.

- When the exit block was completely signed off, it showed that all of the test requirements for that block had been met.

4.3 Benefits and Lessons Learned from OTP and Test Procedure Development

The TPT paradigm complemented with a TPT/OTP_EXECUTE procedural template provided a fairly unique efficiency: test procedures could be almost completely written and reviewed without being impacted by delays caused by negotiations on specific test details. Because those specific test details are contained in the TPT generated OTP, the 
procedure can step through the OTP execution sequence for a set of OTP IDs even while there are TBDs still contained within those OTPs.

A general lesson learned from the test and procedure development phase was that the effort invested in generating the products necessary to prepare for the start of the test became an essential part of being prepared to make adaptations to OTPs and the procedure during testing. At the very start of the cryo-vac campaign, we already had established trust between stakeholder teams, we shared a common vocabulary for the types of changes that might be necessary, and we had a mutual understanding of capabilities and constraints on the TPT and instruments.

\subsection{OTP Execution Overview}

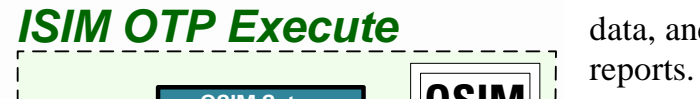
ISIM OTP Execute

Figure 5-1 Top Level TPT and OTP EXECUTE Flow

\section{OPTICAL TEST EXECUTION}

Figure 5-1 depicts a top level flow of the actions that occur starting with the OTP delivery by the Test Planner to a completed optical test. The test planner delivers the test shortly before OTP execution to make sure that the current TPT calibrations and database references are used in the OTP. The TPT Produces various outputs that maintain traceability, assist in interpreting

When the OTP is executed by the ISIM OTP EXECUTE() script, each test activity is handled incrementally with a standardized sequence of steps. First, the parameters associated with any specified ISIM or OSIM re-configuration are sequentially (by instrument) parsed, checked, and then passed to the flight scripts that execute the reconfiguration. Once ISIM and OSIM are in the desired configuration, OTP_EXECUTE enters the exposure block.

In the exposure block, the commands to fire sources and trigger exposures are executed such that the timing of the source and exposure are as repeatable as possible. If multiple exposures in the identical configuration are desired, the exposure block can be repeated as desired. Once exposures are complete, a block of clean-up activities are run, such as extinguishing any source that is not configured to remain on during the next activity. Once one activity is complete, OTP_EXECUTE runs through the same process with the next activity until the entire OTP is completed.

\subsection{Optical Test Operations}

The optics team staffed two positions 24/7 during the ISIM Cryo-Vac Tests: an Optics Test Planner (TP) and a QuickLook Analyst (QLA). For each OTP, the nominal delivery and execution process is shown in Figure 5-2.

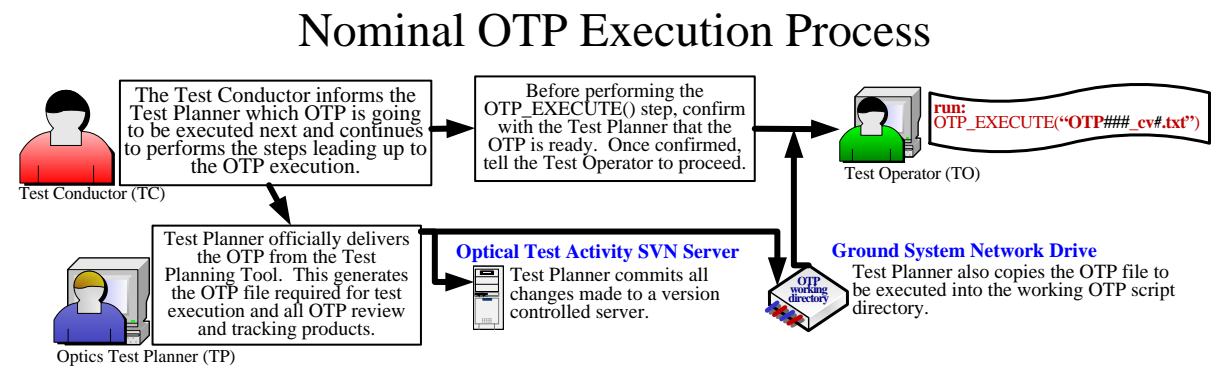

Figure 5-2 Nominal OTP Execution

At the outset of testing, most pre-planned OTP did not produce the desired result. However, once the re-calibration of TPT models were completed based on the initial testing, there were several long stretches of continuous successful OTP executions. One test conductor even remarked that his shift was awfully boring because they just watched a long OTP run without any issues for eight hours. However, we still typically ended up executing two to three times more OTPs that 
had been initially planned. That means a lot of the as-run procedure ended up being blackline insertions of OTPs; however, we established a process that made it possible to turnaround a revised OTP in as little as 5 minutes without compromising QA, QC, traceability, etc. This process is diagrammed in Figure 5-3.

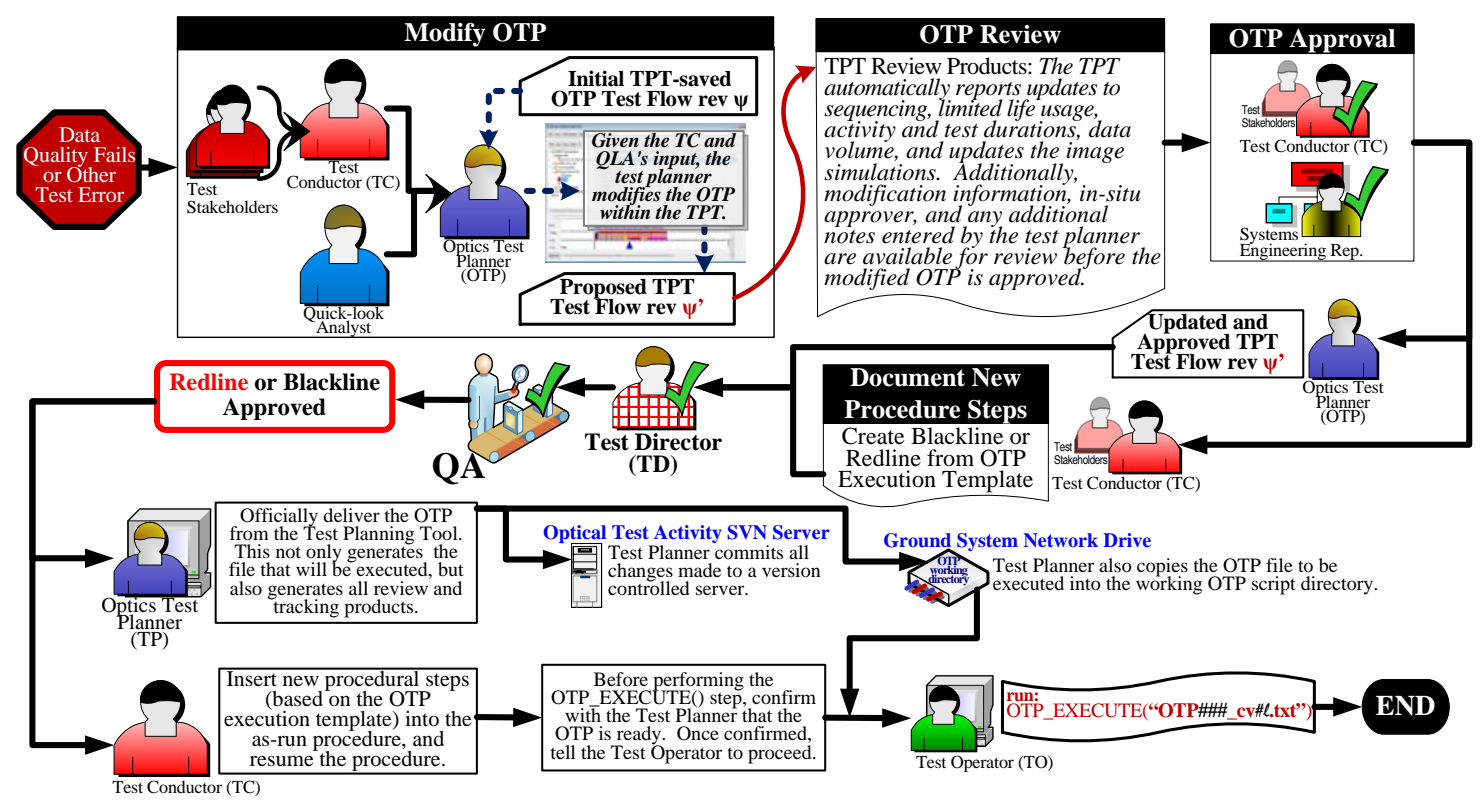

Figure 5-3 Procedure for Implementing a new OTP

\subsection{Logging Results and Reporting Status}

Keeping track of an optical test campaign that runs $24 / 7$ for weeks or months is very challenging. The amount of information is overwhelming, and logging and transferring that information to analysts and folks working on subsequent shifts was a recognized problem during the second ISIM cryo-vac test. The ISIM Optics Test Planning team saw an opportunity to extend the utility of the test planning tool to help alleviate some of the issues logging and communicating test status and issues. First, at a shift change, a test planner would generate an OTP test report. The report would include information about the OTPs run in the past 9 hours, and show the OTPs that were scheduled to run in the next 9 hours.

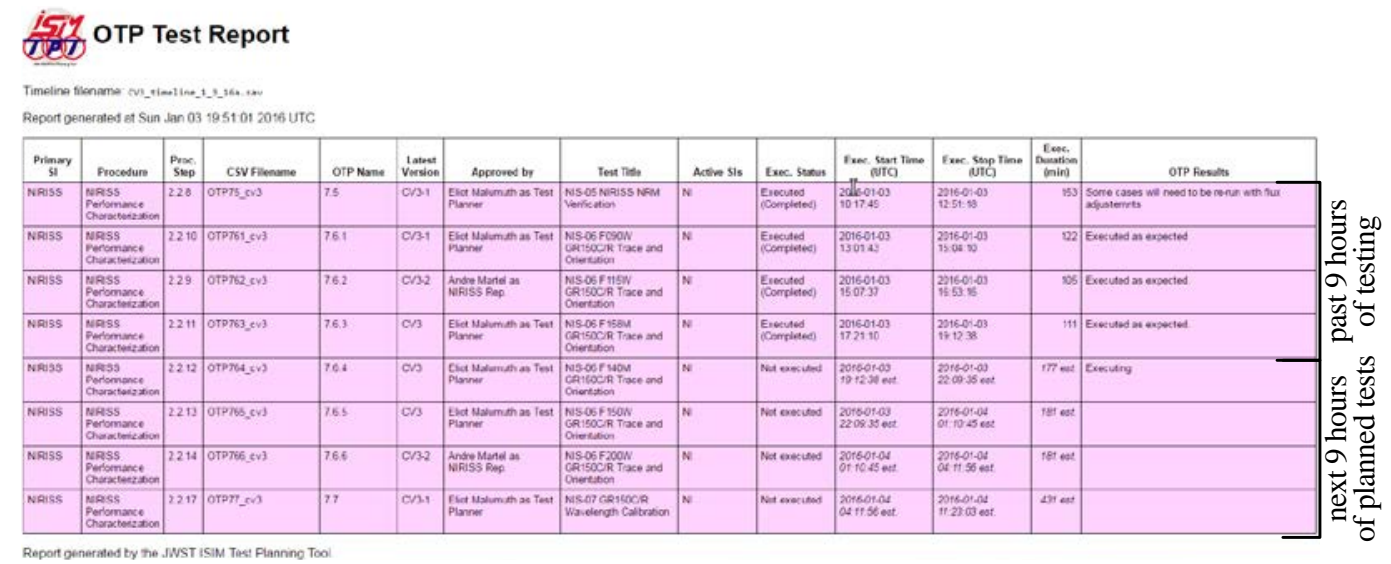

Figure 5-4 OTP Test Report for Shift Change

Additionally, the addition of the OTPInfo GUI incorporated logging the outcome of the OTP execution into the TPT so that all of the OTP information was consolidated in one location. That also allowed for a Test Planning Tool feature to check the OTP_EXECUTE() logs and automatically populate OTP timing information and whether the OTP completed all planned activities. The complete record of an OTP can then be exported from the OTPInfo GUI in HTML or as a PDF. So at the end of the third ISIM cryo-vac tests, a detailed report could be produced for any one of the 693 OTPs executed. 


\section{CONCLUSION}

From an organizational standpoint, using a numerical ID to help delineate the end-to-end linkage between a sciencederived optical requirement to an optical test script that is run in the laboratory to an analysis work plan and product is an elegant tool that greatly assisted us in dealing with our very complex program.

From an implementation standpoint, implementing a TPT to "bridge the gap" between operations scripting and written optical test plans and procedures was invaluable to navigating our lengthy and complex test program. We designed the TPT in a modular manner --- i.e., it was designed "for change," knowing that the software interface to operations commanding and even SI hardware might change during I\&T. We designed it to comply with flight standards of QA, CM, hardware safety, and traceability, but with the flexibility to accommodate the rapid adjustments needed to accomplish an optical test.

We have presented an overview of many of the methods that we used to accomplish ISIM CV testing and verification, from the early organization of requirements to the execution of test scripts and later data analysis. We have also described the motivations and forces that drove us to our test planning, execution, and analysis architecture. Our effort was highly successful and we hope that other workers might consider similar organizational, test, and analysis approaches for their future programs.

\section{ACKNOWLEDGEMENTS}

The authors gratefully acknowledge the contributions of optical, mechanical, electrical, and systems engineers, managers, and scientists associated with the JWST project, the ISIM element, the Science Instruments and their partners [i.e., FGS Guider and NIRISS, provided by the Canadian Space Agency (CSA) and Honeywell; MIRI, provided by the European Consortium with the European Space Agency (ESA), and by the NASA Jet Propulsion Laboratory (JPL); NIRCam, provided by the University of Arizona and Lockheed Martin; and NIRSpec, provided by ESA, with components provided by GSFC], and OSIM.

We acknowledge the leadership of P. Davila (GSFC, retired) and B. Bos (GSFC) in the development of requirements, their organization, and the TPT effort and strong support from J. Dunn (GSFC).

We dedicate this paper to the memory of B. Greeley. His many contributions to the development of OSIM and ISIM were vital to our success.

This work is supported by the James Webb Space Telescope project and NASA Goddard Space Flight Center.

\section{REFERENCES}

${ }^{1}$ Rieke, G. H. et al., “The Mid-Infrared Instrument for the James Webb Space Telescope, I: Introduction,” PASP Vol. 127, Issue 953, 584--594 (2015)

${ }^{2}$ Bagnascoa, G. et al., "Overview of the Near Infrared Spectrograph (NIRSpec) Instrument on-board the James Webb Space Telescope (JWST)”, Proc. SPIE 6692, 66920M (2007)

${ }^{3}$ Rieke, M., Kelly, D. and Horner, S., “Overview of James Webb Space Telescope and NIRCam's Role,” Proc. SPIE 5904, 1--8 (2005).

${ }^{4}$ Doyon, R. et al., "The JWST Fine Guidance Sensor (FGS) and Near-Infrared Imager and Slitless Spectrograph (NIRISS),” Proc. SPIE 8442, 84422R (2012)

${ }^{5}$ Greenhouse, M. A. et al., "Status of the James Webb Space Telescope science instrument payload,” Proc. SPIE 9904, in press (2016)

${ }^{6}$ Kimble, R. A. et al., “Cryo-vacuum testing of the JWST Integrated Science Instrument Module,” Proc. SPIE 9904, in press (2016)

${ }^{7}$ Sullivan, J. F. et al., "JWST's optical telescope simulator for verification of the Integrated Science Instrument Module," Proc. SPIE 9951, in press (2016)

${ }^{8}$ Knight, J. S., “Hartmann test for the James Webb Space Telescope,” Proc. SPIE 9904, in press (2016)

${ }^{9}$ Smith, K. Z., "Calibration results using highly aberrated images for aligning the JWST instruments to the telescope," Proc. SPIE 9904, in press (2016)

${ }^{10}$ Rohrbach, S. O. et al., “ISIM Optical Performance Verification Plan,” JWST-PLAN-018335, internal NASA GSFC publication

${ }^{11}$ Reichard, T. A. et al., "Cryogenic optical test planning using the Optical Telescope Element Simulator with the James Webb Space Telescope Integrated Science Instrument Module,” Proc. SPIE 9951, this volume (2016) 